Tiranas y polacas en la tonadilla escénica: poesía, música y baile en los escenarios teatrales de la corte madrileña

Sara Navarro

Università Europea di Roma (Italia) 



\title{
Tiranas y polacas en la tonadilla escénica: poesía, música y baile en los escenarios teatrales de la corte madrileña
}

\section{Tyrants and Polish in the scenic tonadilla: poetry, music and dance in the theatrical stages of the Madrid court}

\author{
Sara Navarro \\ Università Europea di Roma (Italia) \\ lalandasara.navarro@unier.it
}

Fecha de recepción: 19 de septiembre de 2019

Fecha de aceptación: 20 de octubre de 2020

\section{Resumen}

La investigación que presentamos pretende ser un acercamiento a las características propias de la tirana y la polaca insertas en las tonadillas escénicas más representativas de los escenarios teatrales de la corte madrileña en la segunda mitad del siglo XVIII y principios del siglo XIX. Se realizará un estudio de autoría, que nos introducirá en el momento de mayor auge de esta tipología de piezas y a su utilización por parte de los compositores de la época. Asimismo, se trazará un marco comparativo del número de intérpretes de estas piezas en relación con el resto de números de la tonadilla y se cotejarán, a modo evolutivo, las particularidades poético-musicales y de representación escénica; características que nos permitirán observar si las peculiaridades expuestas por tratadistas en relación con la tirana y la polaca coinciden o son modificadas al insertarse estas composiciones en la tonadilla escénica.

Palabras clave: Tiranas (música); Polacas (música); Tonadilla escénica; Catálogos; Tratadistas; Patrimonio cultural español.

\begin{abstract}
The research we present aims to be an approach to the characteristics of the tiranas and the polacas (musical genres) inserted in the most representative stage tonadillas of theatrical stages of the Madrid court in the second half of the eighteenth century and early nineteenth century. An authorship study will be carried out, which will introduce us at the moment of greatest boom of this type of pieces and its use by the composers of the time. Likewise, a comparative framework of the number of interpreters of these pieces will be drawn in relation to the rest of the numbers of the tonadilla and the poetic-musical particularities and scenic representation will
\end{abstract}


Tiranas y polacas en la tonadilla escénica... - Sara Navarro

be checked in an evolutionary way; characteristics that will allow us to observe if the peculiarities exposed by treatists in relation to the tyrant and the polish coincide or are modified when these compositions are inserted in the scenic tonadilla.

Keywords: Tiranas (musical genre); Polacas (musical genre); Scenic tonadilla; Catalogs; Treaters; Spanish Cultural Heritage.

\section{APROXIMACIÓN A LOS ESTUDIOS SOBRE LA TODADILLA ESCÉNICA}

El intermedio de las comedias resultó ser uno de los momentos más esperados de la escena teatral española del siglo XVIII y principios del siglo XIX. La tonadilla escénica, con su aire nacional de cantes y bailes de contenido folclórico y popular, atrajo la atención del público de los teatros de corte, en particular de los escenarios madrileños del Príncipe y la Cruz.

Este interés fue recogido por investigadores de época como Barbieri (19861988) o Pedrell (1897-1898); estudioso, este último, que profundizó en el teatro lírico español anterior al siglo XIX, analizando el género de la tonadilla como pilar de lo regional y del folclore español.

José Subirá (1928-1930, 1932, 1933) recogería el testigo como musicólogo realizando los primeros estudios monográficos sobre este género así como el Catálogo de la Sección de Música de la Biblioteca Municipal de Madrid (1965), que posibilitó no solo conocer las tonadillas que se encuentran en esta biblioteca sino, asimismo, permitió comprobar tanto las partes de que consta cada tonadilla y sainete como los personajes y comediantes partícipes.

Tras estas investigaciones tan solo encontramos estudios aislados a finales de siglo XX como los de Julio Gómez (1986), Luis Iglesias de Souza (1991-1996), Antonio Álvarez Cañibano (1996) y alguna publicación en forma de edición crítica de Cabañas Alamán (Esteve, 1992) y Suárez Pajares (Esteve et alt., 1998). Habrá que esperar a inicios del presente siglo para encontrar estudios con una mayor profusión e interdisciplinaridad, frutos del trabajo de equipos de investigación y proyectos de musicólogos como Begoña Lolo (2002, 2003) y Germán Labrador (2003), incluso con trabajos conjuntos (2005) así como investigaciones realizadas por la entonces directora de la Biblioteca Histórica Municipal de Madrid, Ascensión Aguerri (2003). Aunque, como expone la especialista en teatro musical Aurèlia Pessarrodona i Pérez (2015), la interdisciplinaridad en el estudio de la tonadilla se desarrolló especialmente a través del diálogo abierto y actas del seminario "Teatro y música en la época de Manuel García" (Cádiz, 18-21 de noviembre de 2004) y el congreso "Teatro y música en España: los géneros breves en la segunda mitad del siglo XVIII" (Madrid, 3-10 de marzo de 2007). Estos encuentros difundieron la importancia del teatro lírico musical con unas perspectivas de amplio alcance que condujeron a una 
apertura en la óptica de estudio y a un interés de autores internacionales como Haidt (2011) y Le Guin (2014).

Retrotayéndonos a las obras citadas con anterioridad de Subirá (1928-1930, pp. 263-272), es de destacar, pues constituye el punto de partida de la presente investigación, el capítulo titulado La "tirana" y la "polaca" como finales tonadillescos, donde se expone, ofreciendo una gran variedad de ejemplos textuales, cómo las seguidillas epilogales fueron sustituidas por tiranas y polacas en el último tercio del siglo XIX.

Entre las investigaciones realizadas sobre la tirana como forma poético-musical es necesario destacar el estudio de Subirá publicado en la revista hispánica de teatro Segismundo. La voz de la "tirana" del Diccionario de la Música Española e Hispanoamericana de Mariano Pérez Gutiérrez y Rubén Pérez Bugallo (1999-2002, pp. 305-306) recoge tanto la investigación apenas mencionada como una revisión de los diccionarios de música de la segunda mitad del siglo XIX, entre los que puede mencionarse los de Roca Bisbal (1852), Fargas Soler (1852, p. 207), Melcior (1859, p. 416), Luisa Lacál (1900, p. 538), Michel Brenet (1946, p. 496) o Pedrell (1894, p. 457); además esta voz o mención recoge una serie de fuentes de referencia básicas como son la Colección de seguidillas, coplas, tiranas y polos de Don Preciso (1799) y uno de los primeros textos que estudian la inserción de esta composición en la tonadilla escénica, me refiero a la obra Tonadillas teatrales inéditas de Subirá (1932).

En este mismo marco de referencia, para la elaboración de la presente investigación hemos analizado los diccionarios de la primera mitad del siglo XX encontrando menciones en el Diccionario de la música ilustrado de Pahissa (ca. 1929, p. 1146) así como en el de Acholes (1964, p. 1248) y Pena y Anglés (1954, p. 2121); textos que han sido estudiados junto a investigaciones más recientes, como la realizada por Faustino Núñez, que nos muestra una posible clasificación de las tiranas en la Guía comentada de música y baile preflamencos (2008).

A diferencia de lo que ocurre con la tirana, las referencias que encontramos sobre la polaca son escasas. Al estudiar la voz o mención "polaca" encontramos dos casuísticas que no guardan relación con el número musical: la primera hace alusión a una de las compañías de teatro (Pena y Anglés, 1954, p. 2121) mientras que la segunda se refiere a la polonesa (Luisa Lacál, 1900, p. 417; Brenet, 1946, p. 414).

\section{PERIODIZACIÓN, CONTEXTUALIZACIÓN Y FUNGIONALIDAD DE LAS TIRANAS Y POLACAS EN LA TONADILLA ESCÉNICA}

Al estudiar el desarrollo evolutivo de la tonadilla con tiranas observamos que este género comienza a estar presente, aunque en número ínfimo, durante el reinado de Fernando VI (1746-1759) y tiene un crecimiento exponencial en el período de Carlos III (1746-1759), momento que podemos definir como de apogeo. Por el 
contrario, el retraimiento del uso de las tiranas deviene durante la dinastía de Carlos IV (1788-1808), que va en contraposición con el momento de esplendor de las polacas al interno de la tonadilla.

Si analizamos esta casuística por décadas, advertimos cómo el período entre 1780-1789 es el que cuenta con mayor número de tiranas en las tonadillas; etapa que coincide con la descrita por Subirá ${ }^{1}$ como de madurez y apogeo. Por otra parte, la fase de esplendor de tonadillas con polacas se ubica, dentro de la clasificación de Subirá, tanto en la etapa de madurez y apogeo como en la de hipertrofia y decrepitud en el campo de las tonadillas.

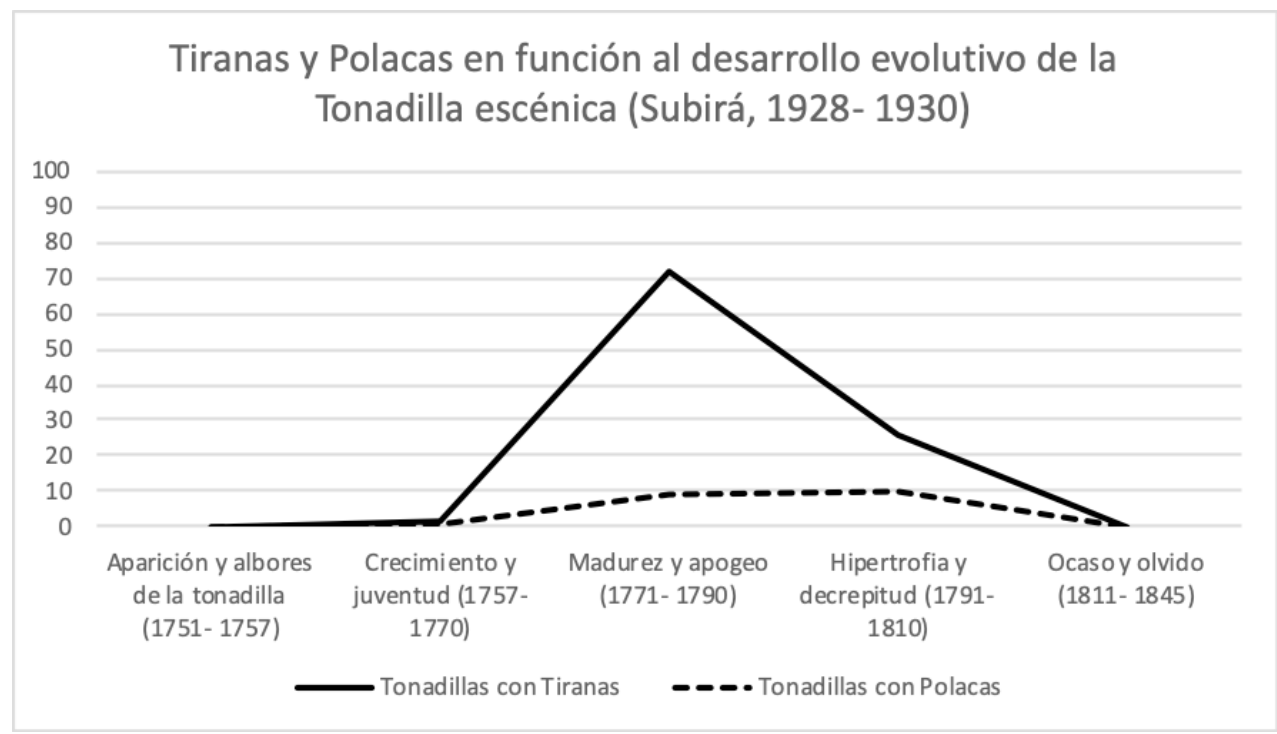

Gráfica 1. Periodización de las tiranas y polacas en las tonadillas escénicas. Fuente de elaboración propia

En la gráfica precedente observamos las décadas en las que existe mayor producción de cada uno de los números estudiados. En relación a las tonadillas con tiranas la década de apogeo (1780-1789) se caracteriza por la presencia de las composiciones de Laserna y Esteve, autores que están en la cúspide en cuanto a composición de tonadillas se refiere.

1 Las cinco fases en las que periodiza el desarrollo evolutivo de las tonadillas Subirá son: Aparición y albores de la tonadilla (1751-1757), Crecimiento y juventud (1757-1770), Madurez y apogeo (1771-1790), Hipertrofia y decrepitud (1791-1810), Ocaso y olvido (1811-1845) (Subirá, 1928-1930, p. 88). 
Por su parte, la producción de tonadillas con polacas refleja su época más fructífera en el período de 1790 a 1809, etapa en la que coinciden los compositores Laserna y Moral como autores destacados del género tonadillesco. Adviértase cómo la predilección que muestra Moral por la polaca, conteniendo una moraleja en relación con el asunto literario de la tonadilla, fue cotejada por Subirá, quien planteó la hipótesis de que pudiera haber sido este autor quien diera carta de naturaleza a la polaca en nuestro país, mientras que Laserna podría haberse dejado influenciar en varias de sus composiciones (1928-1930, p. 272).

La afirmación de Subirá no parece probable ni por la datación de la primera polaca de Moral (1797), fecha muy reciente en comparación con las de Laserna, ni por el número de polacas que Moral en comparación con Laserna compuso, aproximadamente un tercio, como se advierte en la siguiente tabla, que recoge los autores de tonadillas y la presencia de tiranas y polacas en este género:

Tabla 1. Tiranas y polacas de las tonadillas escénicas de la Biblioteca Histórica Municipal de Madrid y Biblioteca Nacional de España. Fuente de elaboración propia

\begin{tabular}{lccc}
\hline Autor & Tonadillas & $\begin{array}{c}\text { Tonadillas con } \\
\text { tiranas }\end{array}$ & $\begin{array}{c}\text { Tonadillas } \\
\text { con polacas }\end{array}$ \\
\hline Anónima & 278 & 6 & 1 \\
\hline ARANAZ, Pedro (Tudela) & 17 & 2 & - \\
\hline BRUZONI, Lorenzo & 3 & 2 & - \\
\hline BUSTOS, Mariano & 21 & 2 & - \\
\hline ESPINOSA & 1 & 1 & - \\
\hline ESTEVE, Pablo & 369 & 44 & 22 \\
\hline LASERNA, Blas de & 710 & 46 & 6 \\
\hline MORAL, Pablo del & 142 & 8 & - \\
\hline REMESSI & 1 & 1 & - \\
\hline ROSALES, Antonio & 148 & 1 & 30 \\
\hline VALLEDOR, Jacinto & 24 & 2 & - \\
\hline TOTAL & 2025 & 115 & \\
\hline
\end{tabular}

Algunos de los estudiosos del escenario tonadillesco de mediados del siglo XVIII y principios del siglo XIX han querido reflejar la vinculación entre la tirana y la seguidilla. Por ejemplo, en el diccionario editado por Brenet, se expone cómo según Adolfo F. Conde de Schack, en su Historia de la literatura y del arte en España (1886) define este baile andaluz como una ligera modificación de las seguidillas (1946, p. 496).

Anglés y Pena, tan solo con una década de diferencia, señala la supresión de la seguidilla a causa de la entrada en escena de la tirana con las siguientes palabras: "Los maestros de la tonadilla escénica la cultivaron hasta el punto de hacer que 
desterrase a la seguidilla" (Anglés y Pena, 1954, p. 2121). Asimismo, Subirá expone que las tiranas, polacas y finales fueron los sucesores de la seguidilla que daban fin a la tonadilla escénica, cuando esta forma epilogal comenzó a causar hastío. En ocasiones, si la tirana se presentaba como número final, análogamente a lo que acontecía a las seguidillas, era continuada por una "coda" formada por un breve número (1928-1930, pp. 263-272). La aparición de esas diversas formas, según el citado autor, no se produjo escalonadamente, ni fue preciso que la una se aboliese para que surgiera la que le había de suceder, ya que ambas se entremezclaban, incluso, en una misma obra.

Si estudiamos detenidamente los números de las tonadillas con tiranas, podemos llegar a la conclusión que el porcentaje de tonadillas que tiene seguidillas al mismo tiempo es bastante significativo, por lo que podría confirmarse la convivencia de ambos números. Por el contrario, si partimos de la consideración de que las tonadillas con polacas suelen enmarcarse mayoritariamente en un período posterior y que el número de tonadillas con polacas que contienen seguidillas podría aproximarse a un cuarto de las mismas, cabría preguntarse si la seguidilla en esta fase dejó de ser protagonista principal de este género y si la polaca suplantó en mayor o menor medida a la seguidilla. En este sentido, Subirá muestra que la polaca puede ser considerada la sucesora de la tirana en la tonadilla escénica, quien a su vez fue la continuadora de la seguidilla. Esta afirmación parece lógica aunque el influjo de la polaca no fue tan hondo como los números anteriores (tirana y seguidilla), quizá por tratarse de un producto extranjero.

En línea con la proposición anterior advertimos que tan solo una tonadilla de las estudiadas contiene conjuntamente una tirana y una polaca, la tonadilla compuesta en 1803 Fin de fiesta de las funciones Reales de Blas de Laserna, que puede definirse como comedia de pasos o folla (Biblioteca Histórica Municipal de Madrid —en adelante BHM-, Mus 642-10). Asimismo, si atendemos al que sería el modelo de estructura de la tonadilla (entable, coplas y seguidillas), podemos afirmar que en el supuesto de suplantar la tirana y la polaca a la seguidilla, su localización preferente sería el número final con o sin la coda ${ }^{2}$, es decir, la denominada seguidilla epilogal (1928-1930, p. 263). El análisis de la siguiente gráfica nos permite contabilizar las tiranas situadas al final de la obra, existiese o no la coda, en un $75 \%$ aproximadamente de los casos, por lo que tendría lógica la hipótesis de Subirá, siendo símil la proporción numérica de las polacas en relación a su localización prevalente como número final de las tonadillas escénicas.

2 En algunas de las tiranas como pieza final encontramos un pequeño número que hace las veces de coda, la cual es simplemente una despedida. 


\section{Localización de Tiranas y Polacas en la Tonadilla escénica}

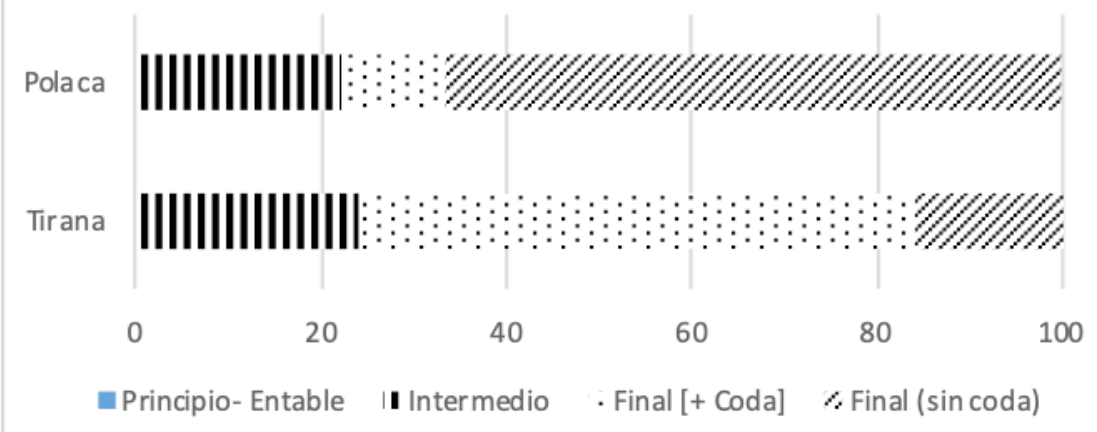

Gráfica 2. Localización de las polacas y tiranas en la tonadilla escénica. Fuente de elaboración propia

En la gráfica precedente se advierte, asimismo, que tanto la tirana como la polaca fueron intermedios en un gran número de ocasiones, localización que también fue frecuente para las seguidillas.

\section{GARACTERÍSTICAS MUSICALES}

La tirana ha sido definida en sus características musicales por diversos tratadistas. Fargas y Soler especifica que es una "canción española en compás ternario, de un movimiento moderado y de un ritmo sincopado" (1852, p. 207). De modo similar, Melcior, en su Diccionario Enciclopédico de la música, dedicado a Hilarión Eslava, expone que la tirana es "una canción española muy graciosa, que se usaba en otro tiempo, la cual tenía un movimiento moderado y semicopado en compás ternario" (1859, p. 416).

Si estudiamos las características musicales de la tirana centrándonos en las indicaciones que más profusamente son propuestas por los estudiosos, es decir, el compás, la agógica y el ritmo, advertimos las consideraciones de musicólogos como Pedrell quien expone que las tiranas fueron en su origen aires de baile y canto de movimiento moderado, exponiendo, a diferencia de los anteriores, que su compás predominante es el 6/8 (1894, p. 457).

A modo de réplica de lo que se ha expuesto del diccionario de Pedrell encontramos el Diccionario de Música ilustrado de Pahissa (ca. 1929, p. 1146) y el Diccionario Oxford de la música. En el último mencionado se señala la posibilidad 
de escritura en 3/4 o en 6/8, su carácter gracioso y moderado además del origen de la denominación a las palabras del estribillo “! Ay, tirana!” (1964, p. 1248). En línea con lo anterior, aunque con alguna que otra matización, el diccionario de Lacál expone, en relación a los compases en que era interpretada la tirana, que su discurso musical se desarrolla en $3 / 4,6 / 8$ o $3 / 8$, con lo que añade una nueva posibilidad métrica, además de indicar que su movimiento es lento (1900, p. 538).

En la tabla del apéndice documental puede observarse que las tiranas insertas en las tonadillas son compuestas en su mayoría en compás de 3/8. Por tanto, sólo el diccionario de Lacál se ajusta a la métrica más frecuente de este número, aunque deben considerarse en menor medida los compases de $3 / 4$ o $6 / 8$, como ya se ha citado con anterioridad (1900, p. 538). Asimismo, tendrá que tenerse en consideración otros compases ternarios en diversidad de subdivisión no citados por los tratadistas, ya que existe un caso en subdivisión binaria, en la tonadilla El remedo de los locos de Aranaz (Biblioteca Histórica Municipal (BHM), Mus 77-21) y tres tiranas en subdivisión ternaria en las tonadillas Los gallegos de la siega (BHM, Mus 117-4), No hay buen estado a disgusto (BHM, Mus 172-15) y El poeta (BHM, Mus 102-9).

Para el análisis de las características musicales de las polacas tenemos que acudir en exclusividad al estudio de los manuscritos musicales, visto que no existen estudios de tratadistas sobre este número. En relación a la métrica, la supremacía en número lo tienen aquellas en $3 / 4$, aunque se pueden encontrar con compases binarios de subdivisión binaria en Fin de fiesta de las funciones reales de Laserna (BHM, Mus 642-10) y en 3/8 en Los secretos de Moral (BHM, Mus 88-2).

Refiriéndonos al carácter y agógica de la tirana y polaca se constata una paulatina estandarización de la terminología ya que conviven adaptaciones y traducciones al idioma castellano de los términos convencionales italianos. En las tiranas podemos entrever cómo los tratadistas la describen con un tempo moderado o lento, hecho que no guarda relación con los datos recogidos en los manuscritos estudiados, ya que el término y con ello la esencia del movimiento suele ser entendida por los compositores bajo la calificación de Allegretto, expresión en alza en lo que a cantidad se refiere; indicación agógica presente igualmente en las polacas aunque advertimos algunas de las mismas de tempo Andante.

La instrumentación de las tiranas insertas en las tonadillas no es un argumento tratado por los tratadistas de la época, ya que tan solo se limitan a especificar su interpretación con la guitarra en las composiciones independientes. En este sentido, el análisis de los manuscritos se ha desarrollado a través de una comparación con las orquestaciones realizadas de forma general en cada una de las tonadillas que contienen tiranas o polacas. Como resultado de este estudio podemos indicar que predominan aquellas tonadillas que mantienen la instrumentación a lo largo de la interpretación, hecho razonable, ya que de esta forma se juega con el factor de economía de medios. Es interesante observar cómo en algunas tonadillas con tiranas 
se produce una supresión de ciertos instrumentos de viento a partir de la década de 1780 , en particular, de los oboes y trompas. En este sentido, debe considerarse como rasgo característico de ciertas tonadillas la sustitución en las tiranas y polacas del oboe por la flauta o flautín, pudiendo ser el mismo músico el que interpretara ambos papeles, ya que están dispuestos en una misma parte en los manuscritos.

Asimismo, aunque como caso particular, debe mencionarse la aparición de la guitarra como único instrumento de acompañamiento de las tiranas en El trueque de los amantes de Laserna (BHM, Mus 147-8) y Aviso a los forasteros y mal uso del idioma de Esteve (BHM, Mus 95-2), tonadillas escritas en la década de 1780 a 1789. Como ya hemos mencionado, esta instrumentación fue la más usual en las tiranas independientes, aportando la guitarra una esencia más popular.

\section{GARACTERÍSTICAS POÉTICO-TEXTUALES}

Es interesante, en el discurso de Pedrell, la descripción ofrecida por Don Preciso sobre las tiranas, quien apunta que se cantaban con coplillas de cuatro versos asonantes de ocho sílabas (1894, p. 457). En lo que se refiere a la estructura, será Subirá quien, aparte de afirmar que consta de una copla formada por cuatro versos octosílabos, exponga que contiene un estribillo de metro variado. Pero más interesante, si cabe, es la denominación de tirana nueva bajo la cual se expone una tirana que conserva la forma propia de la tirana musical mas no la de la tirana literaria, abandonando los versos el corte octosílabo y adoptando el decasílabo; esto sería lo que en la década de 1780-1790 sucedería, aunque de manera inversa, con la seguidilla, la cual mantendría el aspecto de seguidilla en el texto mas no en la música (1928-1930, p. 269).

Si nos adentramos en el análisis de los manuscritos musicales en relación a la métrica (véase el apéndice), observamos que el $90 \%$ de los casos cuentan con versos octosilábicos, que, en ocasiones, se combinan con versos de diversa medida. Es frecuente encontrar versos mixtos de 8 sílabas con otros de 10 o 12 sílabas, forma calificada, como se ha anunciado con anterioridad, de tirana nueva (Subirá, 19281930, p. 269).

En las polacas la métrica prototípica es la versificación heptasílábica, aunque existe, igualmente, mezcolanza en este número, siendo utilizados de forma conjunta versos que oscilan de cinco a diez sílabas.

Centrándonos en la rima tanto de las tiranas como de las polacas insertas en tonadillas, puede observarse que guarda una relación, por lo general, asonante, produciéndose la consonancia, fundamentalmente, a través de la repetición literal de fragmentos textuales, siendo estos acompañados, en algunas ocasiones, de una reproducción del discurso musical. 


\section{TEMÁTICA AUTOREFERENCIAL}

Brenet en su Diccionario de la música histórico y técnico pone en boca de Ricardo Rojas la posible funcionalidad de la tirana en la obra La literatura argentina (1924), afirmando que el nombre que lleva alude a los azares de la zalamería con intención amatoria (1946, p. 496).

En realidad, la temática que utilizan tanto la tirana como la polaca es variada. Faustino Nuñez ha realizado la siguiente clasificación de las tiranas en la Guía comentada de música y baile preflamencos: cantables, con guitarra, de acabar, de extranjeros, con otros géneros musicales, instrumentos, lugares, ay tirana, tirana y andar, con jaleo, triunfo, muerte y resurrección de la tirana, otras tiranas y otras obras que contienen tiranas.

Aunque no nos adentraremos en el estudio de las tipologías de polacas y tiranas en función de su temática, no quisiera desdeñar la fuente informativa que nos ofrecen los textos autoreferenciales; información que analizaremos con cautela ya que podría responder únicamente al interés del compositor.

Para llevar a cabo dicho análisis, en primer lugar, se han analizado a modo comparativo los textos autoreferenciales de las tiranas con las etapas descritas por Subirá en relación a la periodización de la tonadilla escénica. En primer lugar, destacamos el título y argumento de la tonadilla La resurrección de la tirana (1781), la cual expone que "la tirana la enterraron y ha vuelto a resucitar" (BHM, Mus 151-3). Este número se localiza en una tonadilla datada a inicios de la década de los años ochenta (1781), momento de inicio o desarrollo de este número dentro de la tonadilla por lo que parece extraña dicha afirmación. Tan sólo podría explicarse esta mención si se refiriese a que el género independiente estaba cayendo en desuso y que su inserción como número de la tonadilla escénica le permitió encontrar una nueva dimensión.

A través del análisis de los textos autoreferenciales podemos advertir, asimismo, la evolución de un conglomerado de números como el cangrejo, la tirana y la seguidilla, que conviven y confluyen según el gusto del compositor y público del momento. Una relación conflictual de estos números se desarrolla, por ejemplo, con el denominado cangrejo en la tonadilla Los celos iguales (BHM, Mus 115-12), aunque la rivalidad mayor se advierte con el bolero, evolución de las seguidillas (Garrido, 2010), y con las historias del general Malbrú ${ }^{3}$. En línea con lo anterior, entre las tonadillas que hemos analizado con tiranas existen referencias al Malbrú en El desengañado de Laserna, donde uno de los personajes insiste en que no se

3 Malbrú o Mambrú hace referencia al duque de Marlborough (John Churchill) a quien se dedica la canción burlesca tras la batalla de Malplaquet (1709), que enfrentó Gran Bretaña y Francia. La canción se popularizó como canción infantil en Francia y España. Para el estudio del Malbrú en la tonadilla estúdiese Prieto Marugán, José (2018). Malbrú en la tonadilla, la zarzuela y otras músicas. Acotaciones: revista de investigación teatral, n. 40, 2018, pp. 11-30. 
preocupe la tirana que el Malbrú acabará (BHM, Mus 124-5). Esta turbia relación con el Malbrú y, en general, con el gusto por el estilo francés, que parece ser una de las causas por las que nos abandona la tirana, se observa tanto en la tonadilla Hospital del desengaño de Laserna (BHM, Mus 135-4) como en la tirana de La Prueba (BHM, Mus 89-11), aunque en este último caso el mensaje se oculta tras una historia de pobreza y desolación.

Es interesante observar como, por el contrario, en el período de realce del Malbrú, existían números autoreferenciales que enaltecen las tiranas como sucede en Los hidalgos de Medellín (BHM, Mus 189-7) o en El paje galanteador. Por ejemplo, en la última tonadilla mencionada se muestra que los franceses en palabras textuales "se deshacen al escucharla" (BHM, Mus 128-8); expresión favorable que encontramos, asimismo, en la tonadilla Por qué de muchas cosas, en la cual se exclama "vivan los españoles que la tirana inventaron" (BHM, Mus 184-3)4.

En esta confrontación incesante de la tirana con otros números, no puede ser olvidada la tonadilla El peluquero y la modista, en la cual se muestra de forma explícita el posible reemplazo de la tirana con las seguidillas boleras en los siguientes versos:

$$
\begin{aligned}
& \text { "Tiranilla, tú te quejas } \\
& \text { y nadie entiende el mal } \\
& \text { ay, tirana, aunque te mueras } \\
& \text { muy pocos lo sentirán } \\
& \text { pues ya quedan las Boleras } \\
& \text { que tu falta suplirán" }
\end{aligned}
$$

(BHM, Mus 179-17)

Como síntesis de lo observado, puede advertirse que la tirana, una vez que entra en el panorama teatral se asienta fuertemente, establece una relación de igual con la seguidilla y subsiste a los achaques que le trajeron el convivir con las nuevas modas.

En cuanto a la autoreferencialidad de las polacas advertimos tan solo un ejemplo en la tonadilla titulada Cada uno tiene su santo de devoción, aunque no trata de una referencia hacia la polaca sino al teatro como género catártico (BHM, Mus 89-5).

4 Existen otros ejemplos significativos entre los que no queremos excluir el de El encuentro de la apasionada, donde se compara la tirana a un potaje nuevo y es considerada de la Italia el hechizo, la sal y primor (BHM, Mus 102-20). 
Tiranas y polacas en la tonadilla escénica... - Sara Navarro

\section{ANÁLISIS DE LOS PERSONAJES}

El análisis de los personajes en las tiranas y polacas nos aporta diversas consideraciones. En primer lugar, se observa cómo las tiranas y polacas no suelen utilizar todos los personajes de la tonadilla. En este sentido, es evidente la reducción en el número de personajes que la interpretan, predominando los dúos en cuanto a las tiranas mientras que las polacas suelen ser números a solo.

Tabla 2. Personajes en las tiranas y polacas en correspondencia con las tonadillas escénicas

\begin{tabular}{|c|c|c|c|c|}
\hline \multirow[b]{2}{*}{ Personajes } & \multicolumn{2}{|c|}{ Tonadillas con tiranas* } & \multicolumn{2}{|c|}{ Tonadillas con polacas* } \\
\hline & Tonadilla & Tirana & Tonadilla & Polaca \\
\hline A solo & 8 & 18 & 5 & 7 \\
\hline A dúo & 23 & 37 & - & - \\
\hline A tres & 32 & 20 & 3 & 2 \\
\hline A cuatro & 15 & 8 & - & - \\
\hline General* & 17 & - & 1 & - \\
\hline Todos & - & - & - & - \\
\hline Incompletas & - & 12 & - & - \\
\hline Total & \multicolumn{2}{|c|}{95} & \multicolumn{2}{|c|}{9} \\
\hline
\end{tabular}

*Las tiranas y polacas analizadas son exclusivamente aquellas que en la partitura cuentan con el calificativo al inicio del número.

Si relacionamos el número de personajes con la datación cronológica, se percibe que el período de mayor producción de tonadillas generales con tiranas fue la década de 1780-1789. En cambio, las tonadillas a solo se encuentran en su mayor apogeo y casi en igual proporción en los decenios de 1780-1789 y 1790-1799.

En comparación con estos datos, encontramos que la única tonadilla general con polaca, Fin de fiesta de las funciones reales de Laserna (BHM, Mus 642-10), es de principios del siglo XIX y que este número predomina en las tonadillas a solo en la primera década del siglo XIX, aunque también es cuantioso en la última década del siglo XVIII.

\section{REPRESENTACIÓN ESCÉNICA}

Una de las cuestiones que plantea mayor incógnita al estudiar la tirana en la tonadilla escénica es la escenografía. En el discurso de Pedrell (1894, p. 457) se ofrece una descripción de Don Preciso, quien apunta que la tirana, al paso que se cantaba con coplillas de a cuatro versos asonantes de ocho sílabas, se bailaba con un compás claro y demarcado, haciendo diferentes movimientos a un lado y otro con el cuerpo. Asimismo anota que las mujeres llevaban un gracioso juguete con el delantal 
al compás de la música al paso que los hombres manejaban su sombrero o el pañuelo a semejanza de las nociones que conservamos de los bailes de las antiguas gaditanas.

Este número, que en sus orígenes se bailaba, según Pedrell, habiendo degenerado el baile en libertinaje, fue desterrado de los saraos. Sin embargo, Don Preciso añade, según expone Pedrell en sus escritos, que "bajo el nombre general de tirana siguieron los aficionados y músicos componiendo multitud de canciones para la guitarra... que pasaron a Petersburgo, Viena y otras cortes, donde el célebre maestro español D. Vicente Martín hizo fanatismo insertándolas en sus óperas" (1894, p. 457). Esta misma afirmación la encontramos en Pedrell, quien presenta cómo el baile cayó en desuso, conservándose solo como canción que solía nombrarse con alguna palabra del estribillo como tirana de la Caramba (1894, p. 457).

Subirá, por su parte, nos ofrece la afirmación de que se incrustaron en la tonadilla escénica desprovistas de todo carácter coreográfico, utilizando tan solo su molde musical para construir números en los que se solía poner a prueba las facultades vocales de los intérpretes.

Si estudiamos detenidamente las indicaciones marcadas en los manuscritos de las tiranas insertas en tonadillas escénicas, podemos observar que en un número apreciable de las mismas se encuentran referencias que pueden conducir a la conclusión de que eran números bailables, ya sea en parte o en su totalidad. Entre las tiranas que poseen coreografías parciales encontramos tanto la tonadilla Los galanes de la Pretola de Esteve, que apunta "bailando los dos concluirse; y ellos hacen lo propio" (BHM, Mus 142-9) como El huésped y la posadera de Esteve en que se precisa la escenografía "se levantan y bailan" (BHM, Mus 115-4).

Es, a su vez, significativo como el baile en algunas de estas tonadillas se presenta como elemento de novedad ante la repetición musical como ocurre en $E l$ desvalido y protegido de Esteve con la anotación "la $2^{\mathrm{a}}$ vez bailan los dos" (BHM, Mus 115-4); mientras que en otras ocasiones el baile es el elemento principal que se busca ensalzar como sucede en Las provincias españolas unidas de Laserna, tal y como se deduce de la indicación "versos y repiten para bailar" (BHM, Mus 189-3);

Algunas de estas anotaciones llevan, incluso, expuesto el nombre de los intérpretes o personajes, como es el caso de El celoso chasqueado de Esteve, en la cual, al final de la tirana se presenta la indicación "Baila Aldobera" (BHM, Mus 141-11) o Los contrabandistas de Laserna, obra en que se indica la expresión "como baila Miguel" (BHM, Mus 158-7). En este sentido, no podemos olvidarnos de cómo en Los Hidalgos de Medellín encontramos, además de la anotación "bailando" mostrada junto al calificativo "tirana del trípili", la expresión "questa tirana se canta y se baila" (BHM, Mus 189-7). Estas anotaciones, en ocasiones, presentan además referencias a instrumentos musicales de pequeña percusión que acompañaban al baile como sucede en El capitán y los negritos de Esteve, que expresa "bailan los dos con sonajas" (BHM, Mus 142-13). 


\section{GONGLUSIONES}

Como resultado del presente estudio puede verificarse que no toda la información de tratadistas es acertada ni completa en lo que a tirana inserta en tonadilla respecta, ni en relación a las características musicales, en el análisis comparativo de textos de los números en correspondencia con las etapas de Subirá, ni en lo que a personajes, instrumentación y coreografía utilizados se refiere.

Desde el análisis de los manuscritos las tiranas pueden describirse como un número de las tonadillas escénicas, que tuvo su florecer y expansión desde la década de 1780 a 1789 , de temática variada, aunque predominantemente amoroso, con versos octosílabos, en ocasiones, entremezclados con otras métricas $(6,9,10,11$, 12 sílabas) y rima asonante. Se trata de una composición musicada principalmente en 3/8 con carácter Allegretto, interpretada por un número variable de actoresmúsicos que la cantaban y bailaban tocando instrumentos de pequeña percusión con un acompañamiento orquestal propio de la tonadilla, que en ocasiones, preveía la sustitución o supresión de instrumentos de viento, o, incluso, la instrumentación de la guitarra que propiciaba a la pieza una esencia más popular.

Por el contrario, es necesario destacar que la ausencia de documentación sobre la polaca nos ha llevado a la necesidad de estudiar este número casi en su totalidad a través de los manuscritos musicales. Las polacas analizadas describen un número que tuvo su expansión en la última década del siglo XVIII y la primera década del siglo XIX, de temática variada, con versos heptasílabos, aunque en ocasiones, usan métricas de 5 a 10 sílabas y con rima predominantemente asonante. Esta composición es, generalmente, musicada en $3 / 4$ o $3 / 8$, con una agógica diferenciada según el número, que contempla tanto un tempo Allegretto como Andante. Es interpretada por un número reducido de actores-músicos (a solo o dúo), que la cantaban con un acompañamiento orquestal propio de la tonadilla, aunque con la sustitución o supresión de los instrumentos de viento.

El estudio de la relación seguidilla-tirana-polaca nos ha permitido reafirmar la teoría de Subirá en relación a la lucha de poder entre los números expuestos, aun considerando su convivencia en algunas tonadillas escénicas. En este sentido, la tirana suplanta la seguidilla como número epílogal de la tonadilla, aunque no podemos olvidarnos de la existencia de seguidillas intermedias en tonadillas con tiranas. Asimismo, se verifica que la polaca es considerada sucesora de la tirana siendo utilizada de número comodín, al ser empleada indistintamente como intermedio o final de la tonadilla escénica. Ambos números, una vez que entraron en el panorama teatral se asentaron fuertemente, tratando hasta su desaparición de sufragar los achaques que le trajeron convivir con nuevas modas. 


\section{REFERENCIAS BIBLIOGRÁFICAS}

Aguerri Martínez, Ascensión (2003). La colección de música y teatro en la Biblioteca Histórica Municipal de Madrid: Apuntes para su estudio. En Lolo, Begoña (comisaria). Paisajes sonoros en el Madrid del siglo XVIII. Tonadilla escénica. Catálogo de la Exposición. Madrid: Ayuntamiento de Madrid, pp. 92-108.

Álvarez Barrientos, Joaquín y Lolo, Begoña (eds.) (2008). Teatro y música en España: los géneros breves en la segunda mitad del siglo XVIII. Madrid: Universidad Autónoma de Madrid.

Álvarez Cañibano, Antonio (1996). La música para teatro. La tonadilla escénica y otros géneros. En La música en tiempos de Goya. Madrid: Centro de Documentación Musical de Madrid, pp. 59-78.

Barbieri, Francisco Asenjo (1986-1988). Legado Barbieri, vols. I y II. En Casares, Emilio (ed.). Madrid: Fundación Banco Exterior.

Don Preciso (1799). Colección de las mejores coplas de seguidillas, tiranas y polos que se han compuesto para cantar a la guitarra. Madrid: [s.n.].

Esteve, Pablo (1992). Tonadillas escénicas a solo, edición de Fernando Juan Cabañas Alamán (ed.). Madrid: Real Conservatorio Superior de Música-Cámara Oficial de Comercio e Industria de Madrid.

Esteve, Pablo et alt. (1998). Tonadillas, edición de Javier Suárez-Pajares. Madrid: ICCMU.

Escritos de Julio Goméz (recopilación y comentarios) (1986). Madrid: Alpuerto.

Garrido, Tomás (2010). Blanco White y la música. Scherzo, año 25, n. 248, pp. 106-113.

Haidt, Rebecca (2011). Women, Work and Clothing in Eighteenth-Century. Oxford: Voltaire Foundation.

Iglesias De Souza, Luis (1991-1996). Teatro Lírico Español, 4 vols. A Coruña: Diputación Provincial.

Labrador López de Azcona, Germán (2003). Una mirada sobre la tonadilla: Música, texto e intérpretes al servicio de un nuevo ideal escénico. En Lolo, Begoña (comisaria). Paisajes sonoros en el Madrid del siglo XVIII. Tonadilla escénica. Catálogo de la Exposición. Madrid: Ayuntamiento de Madrid, pp. 38-48.

Le Guin, Elisabeth (2014). The Tonadilla in Performance. Lyric Comedy in Enlightenment. Spain, Berkeley/Los Angeles/Londres: University of California Press.

Lolo, Begoña (2003). Itinerarios musicales en la tonadilla escénica. En Lolo, Begoña (comisaria). Paisajes sonoros en el Madrid del siglo XVIII. Tonadilla escénica. Catálogo de la Exposición. Madrid: Ayuntamiento de Madrid, pp. 14-32.

Lolo, Begoña y Labrador, Germán (2005). La música en los teatros de Madrid I. Antonio Rosales y la tonadilla escénica. Madrid: Alpuerto. 
Tiranas y polacas en la tonadilla escénica... - Sara Navarro

Ensemble Elyma (2003). El maestro de baile y otras tonadillas. En Garrido, Gabriel (dir.). Harmonia Mundi, K617.

Lolo, Begoña (2002). La tonadilla escénica, ese género maldito. Revista de Musicología, vol. XXV, n. 2, pp. 439-470.

Núñez, Faustino (2008). Guía comentada de música y baile preflamencos (1750-1808). Barcelona: ediciones carena.

Pedrell i Sabaté, Felipe (1897-1898). Teatro Lírico anterior al siglo XIX. Documentos para la Historia de la Música española, 5 vols. La Coruña: Canuto Berea y Compañía.

Pessarrodona i Pérez, Aurèlia (2015). La tonadilla hoy: tres visiones desde el extranjero. Revista internacional d'Art, n. 9, pp. 179-186.

Prieto Marugán, José (2018). Malbrú en la tonadilla, la zarzuela y otras músicas. Acotaciones: revista de investigación teatral, n. 40, pp. 11-30.

Romero Ferrer, Alberto y Moreno Mengíbar, Andrés (eds.) (2006). Manuel García: de la tonadilla escénica a la ópera española (1775-1832). Libro fruto del seminario “Teatro y música en la época de Manuel García (Cádiz, 18-21 de noviembre de 2004). Cádiz: Universidad.

Subirá, José (1928-1930). La tonadilla escénica, 3 vols. Madrid: Tipografía de Archivos.

Subirá, José (1932). Tonadillas teatrales inéditas: libretos y partituras, con una descripción sinóptica de nuestra música lírica. Madrid: Tipografía de Archivos.

Subirá, José (1933). La tonadilla escénica. Barcelona: Labor.

Subirá, José (1965). Catálogo de la Sección de Música de la Biblioteca Municipal de Madrid, vol. I. Madrid: Artes Gráficas Municipales.

\section{DICGIONARIOS}

Acholes, Percy A. (ed.) (1964). Diccionario Oxford de la música. Buenos Aires: Sudamericana, [Bartolome U. Chiesino].

Brenet, Michel (ed.) (1946). Diccionario de la música histórico y técnico. Barcelona: Iberia. Joaquín Gil.

Casares, Emilio (dir.) (1999-2002). Diccionario de la Música Española e Hispanoamericana, 10 vols. Madrid: SGAE.

Fargas y Soler, Antonio (ed.) (1852). Diccionario de la música. Barcelona: Imprenta de Joaquin Verdaguer.

Lacál, Luisa (ed.) (1900). Diccionario de la música: técnico, histórico, bio-bibliográfico. Madrid: (pasaje de la Alhambra, 1), Est. tip. de San Francisco de Sales.

Melcior, C. J. (ed.) (1859). Diccionario enciclopédico de la música. Lérida: Imprenta de Alejandro García. 
Pahissa, Jaime (dir.) [ca.1929]. Diccionario de la música ilustrado. Barcelona: Central Catalana de Publicaciones, Imp. Romana F. Borrés.

Pedrell, Felipe (ed.) (1894). Diccionario técnico de la música. Barcelona: Isidoro Torres.

Pena, Joaquín y Anglés, Higinio (ed.) (1954). Diccionario de la música labor. Barcelona: Lábor, S.A., Tall. Graf. Ibero-Americanos.

Roca Bisbal, Juan Bautista (ed.) (1852). Gramática musical. Barcelona: [s.n.]. 
Tiranas y polacas en la tonadilla escénica... - Sara Navarro

\begin{tabular}{|c|c|c|c|c|c|c|c|}
\hline TÍTULO & AUTOR & AÑO & ORQUESTACIÓN TIRANA & COMPÁS & PERS. & \begin{tabular}{|l|} 
ESTRUCTURA \\
TEXTUAL
\end{tabular} & MSS. \\
\hline Remedo de los locos (El)* & \begin{tabular}{|l} 
ARANAZ, \\
Pedro
\end{tabular} & 1769 & Voz y Bajo & $3 / 4$ & A solo & seguidillas & BHM, Mus 77-21 \\
\hline$! O h$, suerte tirana!* & $\begin{array}{l}\text { ARANAZ, } \\
\text { Pedro }\end{array}$ & $\begin{array}{l}{[1750-} \\
1779]\end{array}$ & $\mathrm{VI}, \mathrm{VI}, \mathrm{VII}, \mathrm{VII}$, Bajo & $3 / 8$ & A dúo & seguidillas & BHM, Mus 98-21 \\
\hline Maja de la Cinga (La) & $\begin{array}{l}\text { BRUZONI, } \\
\text { Lorenzo }\end{array}$ & 1795 & Obl, Obll, Tpal, Tpall, VI, VII, Contrabajo & $3 / 8$ & A solo & 8 y 12 sílabas & BHM, Mus 92-21 \\
\hline $\begin{array}{l}\text { Maja pobre y el majo } \\
\text { enamorado (La) }\end{array}$ & \begin{tabular}{|l|} 
BRUZONI, \\
Lorenzo
\end{tabular} & 1794 & $\begin{array}{l}\text { Fl. I, FI, II, Tpa I, Tpa II, VI, VI(D), VII, VII(D), } \\
\text { Contrabajo }\end{array}$ & $3 / 8$ & A dúo & 8 y 5 sílabas & BHM, Mus 106-10 \\
\hline Dama voluble (La) & $\begin{array}{l}\text { BUSTOS, } \\
\text { Mariano }\end{array}$ & 1790 & $\begin{array}{l}\text { Ob I y Fl. I, Ob II y FI II, Tpa I, Tpa } \\
\text { II, VI, VI(D), VII, VII(D), Contrabajo, } \\
\text { Contrabajo(D) }\end{array}$ & $3 / 8$ & A dúo & 8 y 10 sílabas & BHM, Mus 119-7 \\
\hline $\begin{array}{l}\text { Petimetres y el hospiciano } \\
\text { (Los) }\end{array}$ & $\begin{array}{l}\text { BUSTOS, } \\
\text { Mariano }\end{array}$ & 1790 & $\begin{array}{l}\text { Ob I, Ob II, Tpa I, Tpa II, VI, VI(D), VII, } \\
\text { VII(D), Contrabajo }\end{array}$ & $3 / 8$ & A dúo & 8 silabas & BHM, Mus 183-21 \\
\hline Toma de Mahón (La) & ESPINOSA & 1782 & VI, VII, Bajo & $3 / 8$ & A dúo & 8 silabas & BHM, Mus 104-7 \\
\hline \begin{tabular}{|l|} 
Amante apocado y la dama \\
quejosa (El)
\end{tabular} & \begin{tabular}{|l|} 
ESTEVE, \\
Pablo
\end{tabular} & 1784 & Obl, Obll, Tpal, Tpall, VI, VI(D), VII, VII(D) & $3 / 8$ & A dúo & 8 sílabas & BHM, Mus $111-10$ \\
\hline Autor del Parador del Sol & $\begin{array}{l}\text { ESTEVE, } \\
\text { Pablo }\end{array}$ & 1786 & $\begin{array}{l}\text { Ob I, Ob II, Tpal, Tpall, VI, VI(D), VII, VII(D), } \\
\text { Contrabajo }\end{array}$ & $3 / 8$ & A solo & 8 y 15 sílabas & BHM, Mus 142-12 \\
\hline \begin{tabular}{|l|} 
Aviso a los forasteros y mal \\
uso del idioma
\end{tabular} & $\begin{array}{l}\text { ESTEVE, } \\
\text { Pablo }\end{array}$ & [1784] & Guitarra & $3 / 8$ & A solo & 8 silabas & BHM, Mus 95-2 \\
\hline Bolsillo del Gascón (El) & $\begin{array}{l}\text { ESTEVE, } \\
\text { Pablo }\end{array}$ & 1785 & $\mathrm{VI}, \mathrm{VI}(\mathrm{D}), \mathrm{VII}$, Contrabajo & $3 / 8$ & A dúo & 8 silabas & BHM, Mus $180-15$ \\
\hline Capitán y los negritos (EI) & $\begin{array}{l}\text { ESTEVE, } \\
\text { Pablo }\end{array}$ & 1785 & $\begin{array}{l}\text { Ob I-Fl, Ob II-FI, Tpal, Tpall, VI, VI(D), VII, } \\
\text { VII(D), Contrabajo }\end{array}$ & $3 / 8$ & A tres & 8 silabas & BHM, Mus 142-13 \\
\hline Celos iguales (Los)* & \begin{tabular}{|l} 
ESTEVE, \\
Pablo
\end{tabular} & 1783 & $\mathrm{VI}, \mathrm{VI}(\mathrm{D}), \mathrm{VII}$, Contrabajo & $3 / 8$ & A dúo & 8 y 6 sílabas & BHM, Mus $115-12$ \\
\hline $\begin{array}{l}\text { Celoso chasqueado (El) } \\
\text { y Transformaciones de } \\
\text { Peliche el estudiante }\end{array}$ & $\begin{array}{l}\text { ESTEVE, } \\
\text { Pablo }\end{array}$ & 1787 & $\mathrm{VI}, \mathrm{VI}(\mathrm{D}), \mathrm{VII}$, Contrabajo & $3 / 8$ & A dúo & 8 y 6 sílabas & BHM, Mus 141-11 \\
\hline Conducta de los majos (La) & \begin{tabular}{|l|} 
ESTEVE, \\
Pablo
\end{tabular} & 1783 & Ob I, Ob II, VI, VI(D), VII, Contrabajo & $3 / 8$ & A dúo & 8 silabas & BHM, Mus 178-17 \\
\hline Cortejos reñidos (Los) & $\begin{array}{l}\text { ESTEVE, } \\
\text { Pablo }\end{array}$ & \begin{tabular}{|l}
{$[1780-$} \\
$1788]$ \\
{$[1789-$} \\
$1792]$ \\
\end{tabular} & $\begin{array}{l}\text { Ob I, Ob II, Tpal, Tpall, VI, VI(D), VII, } \\
\text { Contrabajo }\end{array}$ & $3 / 8$ & A dúo & 8 silabas & BHM, Mus 107-9 \\
\hline Cucaña (La) & \begin{tabular}{|l|} 
ESTEVE, \\
Pablo
\end{tabular} & 1775 & Hay particellas de Tpa I, Tpa II. & $3 / 8$ & $\begin{array}{l}\text { A solo y } \\
\text { todos }\end{array}$ & 8 silabas & BHM, Mus 70-1 \\
\hline Delirio humano $(\mathrm{EI})^{*}$ & $\begin{array}{l}\text { ESTEVE, } \\
\text { Pablo }\end{array}$ & {$\left[\begin{array}{l}{[1770-} \\
1789]\end{array}\right.$} & $\begin{array}{l}\text { Ob I, Ob II, Tpal, Tpall, VI, VI(D), VII, } \\
\text { Contrabajo }\end{array}$ & $3 / 8$ & A solo & 8 y 12 sílabas & BHM, Mus 168-12 \\
\hline Desvalido y protegido (EI) & \begin{tabular}{|l} 
ESTEVE, \\
Pablo
\end{tabular} & 1780 & Ob I, Ob II, Tpal, Tpall, Vl, VII, Contrabajo & $3 / 8$ & A dúo & 8 silabas & BHM, Mus 115-4 \\
\hline Disputa de las entradas (La) & \begin{tabular}{|l|} 
ESTEVE, \\
Pablo
\end{tabular} & 1788 & $\begin{array}{l}\text { Ob I, Ob II, Tpal, Tpall, VI, VI(D) VII, VII(D), } \\
\text { Contrabajo }\end{array}$ & $3 / 8$ & A tres & 8 sílabas & BHM, Mus 184-8 \\
\hline \begin{tabular}{|l|}
$\begin{array}{l}\text { Enfermo burlado por el } \\
\text { practicante (EI)* }\end{array}$ \\
\end{tabular} & $\begin{array}{l}\text { ESTEVE, } \\
\text { Pablo }\end{array}$ & \begin{tabular}{|l|}
$1776-$ \\
$1778]$ \\
\end{tabular} & $\begin{array}{l}\text { Ob I, Ob II, Tpal, Tpall, VI, VI(2D) VII, } \\
\text { VII(D2), Contrabajo, Contrabajo(D2) }\end{array}$ & $3 / 8$ & $\begin{array}{l}\text { A dúo, } \\
\text { todos }\end{array}$ & 8 silabas & BHM, Mus 134-6 \\
\hline Enhorabuenas (Las) & $\begin{array}{l}\text { ESTEVE, } \\
\text { Pablo }\end{array}$ & 1788 & $\begin{array}{l}\text { Ob I, Ob II, Tpal, Tpall, VI, VI(D) VII, VII(D), } \\
\text { Contrabajo }\end{array}$ & $3 / 8$ & A tres & 8 silabas & BHM, Mus 139-3 \\
\hline Escuela de Garrido (La) & \begin{tabular}{|l|} 
ESTEVE, \\
Pablo
\end{tabular} & 1783 & $\begin{array}{l}\text { Ob I, Ob II, Tpal, Tpall, VI, VI(D) VII, VII(D), } \\
\text { Contrabajo }\end{array}$ & $3 / 8$ & \begin{tabular}{|l} 
A solo o \\
todos)
\end{tabular} & 8 sílabas (2) & BHM, Mus 178-18 \\
\hline $\begin{array}{l}\begin{array}{l}\text { Esterero, la petimetra y el } \\
\text { oficial (EI) }\end{array} \\
\end{array}$ & $\begin{array}{l}\text { ESTEVE, } \\
\text { Pablo }\end{array}$ & $\begin{array}{l}{[1787-} \\
1788]\end{array}$ & $\begin{array}{l}\text { VI, VI(D) VII, VII(D), Contrabajo, } \\
\text { Contrabajo(D) }\end{array}$ & $3 / 8$ & $\begin{array}{l}\text { (A solo o } \\
\text { todos) }\end{array}$ & 8 silabas & BHM, Mus 163-1 \\
\hline Fonda nueva (La) & \begin{tabular}{|l} 
ESTEVE, \\
Pablo
\end{tabular} & [1785] & $\begin{array}{l}\text { Obl, Obll, Tpal, Tpa II, VI, VI(D), VII, VII(D), } \\
\text { Contrabajo }\end{array}$ & $3 / 8$ & $\begin{array}{l}\text { A cuatro y } \\
\text { todos }\end{array}$ & 8 silabas & $\begin{array}{l}\text { BHM, Mus 178-9 } \\
{[178-7]}\end{array}$ \\
\hline Galanes de la Pretola (Los)* & $\begin{array}{l}\text { ESTEVE, } \\
\text { Pablo }\end{array}$ & $\begin{array}{l}1782- \\
1794 ?]\end{array}$ & $\mathrm{VI}, \mathrm{VI}(\mathrm{D}), \mathrm{VII}, \mathrm{VII}(\mathrm{D})$, Contrabajo & $3 / 8$ & A tres & 8 silabas & BHM, Mus $142-9$ \\
\hline Galanteo nocturno (El) & $\begin{array}{l}\text { ESTEVE, } \\
\text { Pablo }\end{array}$ & 1788 & $\begin{array}{l}\text { Obl, Obll, Tpal, Tpall, Vl, VI(D), VII, VII(D), } \\
\text { Contrabajo }\end{array}$ & $3 / 8$ & A tres & 8 sílabas & BHM, Mus 185-16 \\
\hline Gallegos de la siega (Los) & \begin{tabular}{|l} 
ESTEVE, \\
Pablo
\end{tabular} & 1783 & $\begin{array}{l}\text { Obl, Obll, Tpal, Tpall, VI, VI(D), VII,VII(D), } \\
\text { Contrabajo }\end{array}$ & $6 / 8$ & dúo & 8 silabas & BHM, Mus 117-4 \\
\hline $\begin{array}{l}2 \text { Garrido enfermo y su } \\
\text { testamento }\end{array}$ & $\begin{array}{l}\text { ESTEVE, } \\
\text { Pablo }\end{array}$ & 1785 & Obl, Obll, Tpal, Tpall, VI, VII, Contrabajo & $3 / 8$ & $\begin{array}{l}\text { Maja (A } \\
\text { solo) }\end{array}$ & 8 silabas & $\begin{array}{l}\text { BHM, Mus 186-2; } \\
\text { BNE, MP/267/9; } \\
\text { BNE, MP/267/10 }\end{array}$ \\
\hline
\end{tabular}


Revista de Humanidades, 41 (2020). p. 37-60. ISSN 1130-5029

\begin{tabular}{|c|c|c|c|c|c|c|c|}
\hline \begin{tabular}{|l|} 
Hidalgos de Medellín (Los) \\
o Don Celedonio y Doña \\
Toribia
\end{tabular} & \begin{tabular}{|l} 
ESTEVE, \\
Pablo
\end{tabular} & 1785 & $\mathrm{VI}, \mathrm{VI}(\mathrm{D}), \mathrm{VII}$, Contrabajo & $3 / 8$ & A solo & 8 silabas & BHM, Mus 189-7 \\
\hline Hidalgos de Medellín & $\begin{array}{l}\text { ESTEVE, } \\
\text { Pablo }\end{array}$ & 1785 & Flautín, VI, VI(D), VII, VII(D), bajo, bajo (D) & $3 / 8$ & A tres & 8 sílabas & BHM, Mus 189-8 \\
\hline Huésped y la posadera (EI) & $\begin{array}{l}\text { ESTEVE, } \\
\text { Pablo }\end{array}$ & 1784 & $\begin{array}{l}\text { Obl, Obll, Tpal, Tpall, VI, VI(D), VII, VII(D), } \\
\text { Contrabajo }\end{array}$ & $3 / 8$ & A dúo & 8 Y 10 sílabas & BHM, Mus 115-14 \\
\hline Lecciones (Las) & $\begin{array}{l}\text { ESTEVE, } \\
\text { Pablo }\end{array}$ & 1780 & VI, VII, Contrabajo (expone violón) & $3 / 8$ & A solo & 8 sílabas & BHM, Mus 114-10 \\
\hline \begin{tabular}{|l|} 
Maja barbera, el mancebo y \\
el payo $(L a)^{*}$
\end{tabular} & $\begin{array}{l}\text { ESTEVE, } \\
\text { Pablo }\end{array}$ & 1784 & $\mathrm{VI}, \mathrm{VI}(\mathrm{D}), \mathrm{VII}, \mathrm{VII}(\mathrm{D})$, bajo & $3 / 8$ & A dúo & 8 silabas & BHM, Mus 119-3 \\
\hline Majos del baile (Los) & $\begin{array}{l}\text { ESTEVE, } \\
\text { Pablo }\end{array}$ & 1780 & - & - & - & - & BHM, Mus 134-9 \\
\hline \begin{tabular}{|l|}
$\begin{array}{l}\text { Molinero, la pescadora y el } \\
\text { pajarero (El) }\end{array}$ \\
\end{tabular} & $\begin{array}{l}\text { ESTEVE, } \\
\text { Pablo }\end{array}$ & 1789 & $\begin{array}{l}\text { Obl, Obll, Tpal, Tpall, Vl, VI(D), VII, VII(D), } \\
\text { Contrabajo }\end{array}$ & $3 / 8$ & A tres & 8 sílabas & BHM, Mus 140-9 \\
\hline $\begin{array}{l}\text { No hay buen estado a } \\
\text { disgusto }\end{array}$ & $\begin{array}{l}\text { ESTEVE, } \\
\text { Pablo }\end{array}$ & 1787 & $\begin{array}{l}\text { Obl, Obll, Tpal, Tpall, VI, VI (D), VII, VII(D), } \\
\text { Contrabajo }\end{array}$ & $6 / 8$ & A tres & 8 sílabas? & BHM, Mus 172-15 \\
\hline Novios y la maja (Los) & $\begin{array}{l}\text { ESTEVE, } \\
\text { Pablo }\end{array}$ & 1784 & $\begin{array}{l}\text { Obl, Obll, Tpal, Tpall, VI, VI(D), VII, VII(D), } \\
\text { Contrabajo }\end{array}$ & $3 / 8$ & A tres & 8 silabas & BHM, Mus 141-5 \\
\hline Operísta fingida (La) & $\begin{array}{l}\text { ESTEVE, } \\
\text { Pablo }\end{array}$ & 1786 & VI, VI(D), VII, VII(D), Contrabajo & $3 / 8$ & A tres & 8 sílabas & BHM, Mus 138-5 \\
\hline Paje galanteador (EI) & $\begin{array}{l}\text { ESTEVE, } \\
\text { Pablo }\end{array}$ & 1777 & $\begin{array}{l}\text { FI I, FI II, Tpal, Tpall, VI, VI(D) VII, VII(D), } \\
\text { Vla, bajo, bajo(D) }\end{array}$ & $3 / 8$ & $\begin{array}{l}\text { (A solo o } \\
\text { a dúo) A } \\
\text { dúo }\end{array}$ & 8 silabas & BHM, Mus 128-8 \\
\hline $\begin{array}{l}\begin{array}{l}\text { Paje petimetre, la tía y los } \\
\text { dos sobrinos (El) }\end{array} \\
\end{array}$ & $\begin{array}{l}\text { ESTEVE, } \\
\text { Pablo }\end{array}$ & 1786 & $\begin{array}{l}\text { Obl, Obll, Tpal, Tpall, VI, VI(D), VII, VII(D), } \\
\text { Contrabajo, contrabajo(D) }\end{array}$ & $3 / 8$ & $\begin{array}{l}\text { (A solo o } \\
\text { todos) }\end{array}$ & 8 sílabas & BHM, Mus 186-8 \\
\hline Peluquero y la modista (EI) & $\begin{array}{l}\text { ESTEVE, } \\
\text { Pablo }\end{array}$ & 1788 & $\begin{array}{l}\text { Obl, Obll, Tpal, Tpall, VI, VI(D), VII, VII(D), } \\
\text { Fagot, Contrabajo }\end{array}$ & $3 / 8$ & $\begin{array}{l}\text { A cuatro y } \\
\text { todos }\end{array}$ & 8 sílabas (2) & BHM, Mus 179-17 \\
\hline Pepillo Lechuza retirado* & $\begin{array}{l}\text { ESTEVE, } \\
\text { Pablo }\end{array}$ & 1785 & $\begin{array}{l}\text { Obl, Obll, Tpal, Tpall, VI, VI(D), VII, VII(D), } \\
\text { Contrabajo }\end{array}$ & $3 / 8$ & A todos & 8 silabas & BHM, Mus $178-10$ \\
\hline Pepín fuera de la cárcel & $\begin{array}{l}\text { ESTEVE, } \\
\text { Pablo }\end{array}$ & 1785 & $\begin{array}{l}\text { Obl, Obll, Tpal, Tpall, VI, VI(D),VII, VII(D), } \\
\text { Contrabajo }\end{array}$ & $3 / 8$ & A dúo & 8 y 10 silabas & BHM, Mus 116-12 \\
\hline $\begin{array}{l}\text { Petimetre y el chusco } \\
\text { andaluz (El) }\end{array}$ & $\begin{array}{l}\text { ESTEVE, } \\
\text { Pablo }\end{array}$ & 1785 & $\mathrm{VI}, \mathrm{VI}(\mathrm{D}), \mathrm{VII}, \mathrm{VII}(\mathrm{D})$, Contrabajo & $3 / 8$ & A cuatro & 8 y 12 silabas & BHM, Mus 180-8 \\
\hline Petronita Correa (La) & $\begin{array}{l}\text { ESTEVE, } \\
\text { Pablo }\end{array}$ & 1787 & VI, VI(D), VII, VII(D), Contrabajo & $3 / 8$ & A solo & 8 sílabas & BHM, Mus 90-17 \\
\hline Recién Casados (Los) & $\begin{array}{l}\text { ESTEVE, } \\
\text { Pablo }\end{array}$ & 1788 & $\begin{array}{l}\text { Obl, Obll, Tpal, Tpall, VI, VI(D),VII, VII(D), } \\
\text { Contrabajo, Contrabajo(D) }\end{array}$ & $3 / 8$ & Todos & 8 silabas & BHM, Mus 145-3 \\
\hline Salida de la comedia (La) & $\begin{array}{l}\text { ESTEVE, } \\
\text { Pablo }\end{array}$ & 1788 & $\begin{array}{l}\text { Obl, Obll, Tpal, Tpall, VI, VI(D), VII, VII(D), } \\
\text { Contrabajo }\end{array}$ & $3 / 8$ & A tres & 8 silabas & BHM, Mus 184-12 \\
\hline Todos alaban su gusto & $\begin{array}{l}\text { ESTEVE, } \\
\text { Pablo }\end{array}$ & 1789 & $\begin{array}{l}\text { Obl, Obll, Tpal, Tpall, Vl, VI(D), VII, VII(D), } \\
\text { Contrabajo }\end{array}$ & $3 / 8$ & A tres & $\begin{array}{l}8 \text { y } 13-12 \\
\text { sílabas }\end{array}$ & BHM, Mus 134-4 \\
\hline \begin{tabular}{|l|} 
Visita de Vicente a la \\
Nicolasa (La)
\end{tabular} & $\begin{array}{l}\text { ESTEVE, } \\
\text { Pablo }\end{array}$ & 1789 & $\begin{array}{l}\text { Tpal, Tpall, VI, VI(D), VII, VII(D), } \\
\text { Contrabajo }\end{array}$ & $3 / 8$ & A dúo & 8 silabas & BHM, Mus 107-3 \\
\hline [Al arma, al arma todos] & $\begin{array}{l}\text { LASERNA, } \\
\text { Blas de }\end{array}$ & $178-$ & $\mathrm{VI}, \mathrm{VI}(\mathrm{D}), \mathrm{VII}, \mathrm{VII}(\mathrm{D})$, Contrabajo & $3 / 8$ & - & - & BHM, Mus 155-13 \\
\hline Amo sorprendido (El) & $\begin{array}{l}\text { LASERNA, } \\
\text { Blas de }\end{array}$ & [1783- & $\begin{array}{l}\text { Obl, Obll, Tpal, Tpall, VI, VI(D), VII, VII(D), } \\
\text { bajo (Tachadas las partes de viento } \\
\text { y bajo) }\end{array}$ & $3 / 8$ & $\begin{array}{l}\text { A dúo y } \\
\text { todos }\end{array}$ & 8 y 13 sílabas & BHM, Mus 148-9 \\
\hline Avaricia castigada $(\mathrm{La})^{*}$ & $\begin{array}{l}\text { LASERNA, } \\
\text { Blas de }\end{array}$ & 1788 & $\begin{array}{l}\text { Obl, Obll, Tpal, Tpall, VI, VI(D), VII, } \\
\text { VII(D), bajo }\end{array}$ & $3 / 8$ & $\begin{array}{l}\text { A dúo y } \\
\text { todos }\end{array}$ & 8 y 12 sílabas & BHM, Mus 147-4 \\
\hline Bandos (Los) & $\begin{array}{l}\text { LASERNA, } \\
\text { Blas de }\end{array}$ & [1783] & $\begin{array}{l}\text { Obl, Obll, Tpal, Tpall, VI, VI(D), VII, } \\
\text { VII(D), bajo }\end{array}$ & $3 / 8$ & $\begin{array}{l}\text { A dúo y } \\
\text { todos }\end{array}$ & \begin{tabular}{|l|}
8 y $10-9$ \\
silabas
\end{tabular} & BHM, Mus 161-2 \\
\hline Bola de gas (La)"2" & $\begin{array}{l}\text { LASERNA, } \\
\text { Blas de }\end{array}$ & $\begin{array}{l}(1783- \\
84)\end{array}$ & $\begin{array}{l}\text { Obl, Obll, Tpal, Tpall, VI, VI(D), VII, VII(D), } \\
\text { Contrabajo ( } 2^{\mathrm{a}} \text { tirana sustituye oboes } \\
\text { por flautas) }\end{array}$ & $3 / 8$ & A solo & 8 y 12 silabas & BHM, Mus 85-7 \\
\hline Caballero majo (EI) & $\begin{array}{l}\text { LASERNA, } \\
\text { Blas de }\end{array}$ & 1793 & $\begin{array}{l}\text { Obl, Obll, Tpal, Tpall, VI, VI(D), VII, VII(D), } \\
\text { Contrabajo, Contrabajo(D) }\end{array}$ & $3 / 8$ & A dúo & 8 silabas & BHM, Mus 108-4 \\
\hline Codiciosos burlados (Los) & $\begin{array}{l}\text { LASERNA, } \\
\text { Blas de }\end{array}$ & 1804 & $\begin{array}{l}\text { Cl, Obl, Obll, Fg, Tpal, Tpall, VI, VI(D), VII, } \\
\text { VII(D), Vla, bajo, bajo(D) }\end{array}$ & |- & - & - & BHM, Mus $154-10$ \\
\hline Cómicos de Méjico (Los) & $\begin{array}{l}\text { LASERNA, } \\
\text { Blas de }\end{array}$ & 1788 & $\mathrm{VI}, \mathrm{VI}(\mathrm{D}), \mathrm{VII}, \mathrm{VII}(\mathrm{D}), \mathrm{Fg}$, Contrabajo & $3 / 8$ & A tres & 8 y 10 sílabas & BHM, Mus 184-7 \\
\hline \begin{tabular}{|l|} 
Contrabandistas (Los). Cada \\
uno con su suerte. Los \\
soldados y contrabandistas
\end{tabular} & $\begin{array}{l}\text { LASERNA, } \\
\text { Blas de }\end{array}$ & (1806?) & $\begin{array}{l}\text { Cl, Flautín I, Flautín II, Tpal, Tpall, VI, } \\
\text { VI(D), VII, VII(D),Vla, Fg, bajo, bajo(D) }\end{array}$ & $3 / 8$ & $\begin{array}{l}\text { A solo y } \\
\text { todos }\end{array}$ & 8 y 10 silabas & BHM, Mus 158-7 \\
\hline Cotorra (La) & $\begin{array}{l}\text { LASERNA, } \\
\text { Blas de }\end{array}$ & \begin{tabular}{|l}
{$[1783-$} \\
$1791]$ \\
$(1787)$ \\
Andioc, \\
670 \\
\end{tabular} & VI, VI(D), VII, VII(D), contrabajo & $3 / 8$ & $\begin{array}{l}\text { A dúo y } \\
\text { todos }\end{array}$ & 8 silabas & BHM, Mus 179-15 \\
\hline Chasco de las negrillas $(\mathrm{EI})^{*}$ & $\begin{array}{l}\text { LASERNA, } \\
\text { Blas de }\end{array}$ & \begin{tabular}{|l}
$1783-$ \\
$1786]$ \\
$(1788)$ \\
Andioc, \\
677 \\
\end{tabular} & $\begin{array}{l}\text { Obl, Obll, Cl, Tpal, Tpall, VI, VI(D), VII, } \\
\text { VII(D), Vla, Fg, bajo, bajo (D) }\end{array}$ & $3 / 8$ & $\begin{array}{l}\text { (A solo y } \\
\text { a dúo) A } \\
\text { dúo }\end{array}$ & 8 y 12 sílabas & BHM, Mus 157-9 \\
\hline
\end{tabular}


Tiranas y polacas en la tonadilla escénica... - Sara Navarro

\begin{tabular}{|c|c|c|c|c|c|c|c|}
\hline Desengañado (El) & $\begin{array}{l}\text { LASERNA, } \\
\text { Blas de }\end{array}$ & 1785 & $\begin{array}{l}\text { Obl, Obll, Tpal, Tpall, VI, VI(D), VII, VII(D), } \\
\text { bajón I, bajón II, bajo }\end{array}$ & $3 / 8$ & A tres & 8 y 10 sílabas & BHM, Mus 124-5 \\
\hline $\begin{array}{l}\begin{array}{l}\text { Despedida de los amantes } \\
\text { (La) }\end{array} \\
\end{array}$ & $\begin{array}{l}\text { LASERNA, } \\
\text { Blas de }\end{array}$ & $\begin{array}{l}1786- \\
1794 ?]\end{array}$ & $\mathrm{VI}, \mathrm{VII}$ & $3 / 8$ & A dúo & $\begin{array}{l}8 \text { silabas- } \\
\text { notas }\end{array}$ & BHM, Mus 114-3 \\
\hline Despreciada (La) & $\begin{array}{l}\text { LASERNA, } \\
\text { Blas de }\end{array}$ & $\begin{array}{l}1783- \\
1786] \\
\end{array}$ & $\begin{array}{l}\begin{array}{l}\text { Fl I, FI II, Tpal, Tpall, VI, VI(D), VII, VII(D), } \\
\text { bajo }\end{array} \\
\end{array}$ & $3 / 8$ & A cuatro? & 8-9 sílabas & $\begin{array}{l}\text { BHM, Mus 126-5; } \\
\text { BNE, MC/3060/5 }\end{array}$ \\
\hline Disputa de la boda (La) & $\begin{array}{l}\text { LASERNA, } \\
\text { Blas de }\end{array}$ & 1786 & $\begin{array}{l}\text { Obl, Obll, Tpal, Tpall, VI, VI(D), VII, VII(D), } \\
\text { bajo, bajo (D) }\end{array}$ & $3 / 8$ & A dúo & 8 sílabas & BHM, Mus 153-3 \\
\hline $\begin{array}{l}\text { Encuentro de la apasionada } \\
\text { (El) o Los majos de la } \\
\text { Carrera }\end{array}$ & $\begin{array}{l}\text { LASERNA, } \\
\text { Blas de }\end{array}$ & 1785 & $\begin{array}{l}\text { Obl, Obll, Tpal, Tpall, VI, VI(D), VII, VII(D), } \\
\text { bajo, bajo(D) }\end{array}$ & $3 / 8$ & A solo & 8 y 10 sílabas & BHM, Mus 102-20 \\
\hline Encuentro del pastor (El) & $\begin{array}{l}\text { LASERNA, } \\
\text { Blas de }\end{array}$ & $\begin{array}{l}1783- \\
1794 ?] \\
\end{array}$ & $\begin{array}{l}\text { Obl, Obll, Tpal, Tpall, VI, VI(D), VII, } \\
\text { VII(D), bajo }\end{array}$ & $3 / 8$ & A tres & 8 y 10 sílabas & BHM, Mus 125-3 \\
\hline \begin{tabular}{|l|}
$\begin{array}{l}\text { Enhorabuena de la Pulpillo } \\
(\text { La })^{*}\end{array}$ \\
\end{tabular} & $\begin{array}{l}\text { LASERNA, } \\
\text { Blas de }\end{array}$ & 1785 & $\begin{array}{l}\begin{array}{l}\text { FI I, FI II, Tpal, Tpall, VI, VI(D), VII, VII(D), } \\
\text { bajo, bajo(D) }\end{array} \\
\end{array}$ & $3 / 8$ & A tres & 8 sílabas & BHM, Mus 123-16 \\
\hline Escuela del interés (La) & $\begin{array}{l}\text { LASERNA, } \\
\text { Blas de }\end{array}$ & $\begin{array}{l}1791- \\
1793]\end{array}$ & $\begin{array}{l}\text { Obl, Obll, Tpal, Tpall, Vl, VI(D), VII, } \\
\text { VII(D), bajo }\end{array}$ & - & - & - & BHM, Mus 84-7 \\
\hline \begin{tabular}{|l|}
$\begin{array}{l}\text { Fin de fiesta de las } \\
\text { funciones Reales* }\end{array}$ \\
\end{tabular} & $\begin{array}{l}\text { LASERNA, } \\
\text { Blas de }\end{array}$ & 1803 & - & $3 / 8$ & A dúo & 8-9-10 sílabas & BHM,Mus 642-10 \\
\hline \begin{tabular}{|l|} 
Francés, italiano y majos o \\
El triunfo de las mujeres
\end{tabular} & $\begin{array}{l}\text { LASERNA, } \\
\text { Blas de }\end{array}$ & 1781 & $\mathrm{VI}, \mathrm{VI}(\mathrm{D}), \mathrm{VII}, \mathrm{VII}(\mathrm{D})$, bajo & $3 / 8$ & A cuatro & 8 silabas & BHM, Mus 151-4 \\
\hline Gitanos y el Hidalgo (Los) & $\begin{array}{l}\text { LASERNA, } \\
\text { Blas de }\end{array}$ & [1794?] & $\begin{array}{l}\text { Obl (sustituido por flautines), Obll } \\
\text { (sustituido por flautines), Tpal, Tpall, VI, } \\
\text { VI(D), VII, VII(D), contrabajo }\end{array}$ & $3 / 8$ & A dúo & 8 sílabas & \begin{tabular}{|l} 
BHM, Mus 174-4 \\
{$[175-4]$}
\end{tabular} \\
\hline $\begin{array}{l}\text { Hidalgo lugareño y la } \\
\text { petimetra (El) }\end{array}$ & $\begin{array}{l}\text { LASERNA, } \\
\text { Blas de }\end{array}$ & \begin{tabular}{|l|}
{$[1783-$} \\
$1793]$ \\
\end{tabular} & $\begin{array}{l}\text { Obl, Obll, Tpal, Tpall, VI, VI(D), VII, } \\
\text { VII(D), bajo }\end{array}$ & $3 / 8$ & A solo & 8-10 sílabas & BHM, Mus 105-4 \\
\hline Hospital del desengaño (EI) & $\begin{array}{l}\text { LASERNA, } \\
\text { Blas de }\end{array}$ & $\begin{array}{l}1792- \\
1794 ?]\end{array}$ & $\begin{array}{l}\text { Obl, Obll, Tpal, Tpall, VI, VI(D), VII, VII(D), } \\
\text { bajo, contrabajo(D) }\end{array}$ & $3 / 8$ & A tres & $\begin{array}{l}8-10-12 \\
\text { silabas }\end{array}$ & BHM, Mus 135-4 \\
\hline Majo celoso (El) & $\begin{array}{l}\text { LASERNA, } \\
\text { Blas de }\end{array}$ & 1796 & $\begin{array}{l}\text { Obl, Obll, Cl, Tpal, Tpall, VI, VI(D), VII, } \\
\text { VII(D),Vla, Fg, bajo, contrabajo }\end{array}$ & $3 / 8$ & A dúo & 8-5-6 sílabas & BHM, Mus 108-10 \\
\hline Majos celosos (Los) & $\begin{array}{l}\text { LASERNA, } \\
\text { Blas de }\end{array}$ & $\begin{array}{l}1780- \\
1791] \\
\end{array}$ & $\begin{array}{l}\text { Obl, Obll, Tpal, Tpall, VI, VI(D), VII, } \\
\text { VII(D), bajo }\end{array}$ & $3 / 8$ & A dúo & 8-10 silabas & BHM, Mus 99-6 \\
\hline Majos descontentos (Los) & $\begin{array}{l}\text { LASERNA, } \\
\text { Blas de }\end{array}$ & 1796 & $\begin{array}{l}\text { Obl, Obll, Tpal, Tpall, VI, VI(D), VII, } \\
\text { contrabajo }\end{array}$ & $3 / 8$ & A dúo & $\begin{array}{l}8-6(12) \\
\text { silabas } \\
\end{array}$ & BHM, Mus 111-4 \\
\hline Marqueses regañones (Los) & $\begin{array}{l}\text { LASERNA, } \\
\text { Blas de }\end{array}$ & $\begin{array}{l}1787 \\
\text { Andioc, } \\
768\end{array}$ & $\mathrm{VII} \mathrm{VI}(\mathrm{D}), \mathrm{VII}-\mathrm{VII}(\mathrm{D})$, bajo & $3 / 8$ & - & - & BHM, Mus 157-8 \\
\hline \begin{tabular}{|l|} 
Mayores[Mayorazgos] \\
necios y los soldados (Los) \\
\end{tabular} & $\begin{array}{l}\text { LASERNA, } \\
\text { Blas de }\end{array}$ & $\begin{array}{l}{[1784-} \\
1792] \\
\end{array}$ & $\begin{array}{l}\text { Obl, Obll, Tpal, Tpall, VI, VI(D), VII, } \\
\text { VII(D), bajo }\end{array}$ & $3 / 8$ & - & - & BHM, Mus 181-18 \\
\hline Miscelánea (La) & $\begin{array}{l}\text { LASERNA, } \\
\text { Blas de }\end{array}$ & {$\left[\begin{array}{l}{[1783-} \\
1793]\end{array}\right.$} & $\begin{array}{l}\text { Obl, Obll, Tpal, Tpall, VI, VI(D), VII,VII(D), } \\
\text { bajo }\end{array}$ & $3 / 8$ & A solo & 8-12 sílabas & $\begin{array}{l}\text { BHM, Mus 82-1; } \\
\text { BHM, Mus 83-20; } \\
\text { BNE, MC/3062/9 }\end{array}$ \\
\hline $\begin{array}{l}\begin{array}{l}\text { Novillo de la tarde del día } \\
\text { de San Isidro (El)* }\end{array} \\
\end{array}$ & $\begin{array}{l}\text { LASERNA, } \\
\text { Blas de }\end{array}$ & 1783 & $\begin{array}{l}\begin{array}{l}\text { Fl I, FI II, Tpal, Tpall, VI, VI(D), VII,VII(D), } \\
\text { bajo }\end{array} \\
\end{array}$ & $3 / 8$ & A cuatro & 8 sílabas & $\begin{array}{l}\text { BHM, Mus 164-5; } \\
\text { BNE, MC/3060/3 }\end{array}$ \\
\hline Novio discreto (El) & \begin{tabular}{|l} 
LASERNA, \\
Blas de
\end{tabular} & 1788 & FI I, Fl II, Tpal, Tpall, VI, VI(D), VII, bajo & $3 / 8$ & A dúo & 8 sílabas & \begin{tabular}{|l|} 
BHM, Mus 110- \\
$16[100-16]$
\end{tabular} \\
\hline Petimetra supuesta (La) & $\begin{array}{l}\text { LASERNA, } \\
\text { Blas de }\end{array}$ & {$\left[\begin{array}{l}{[1791-} \\
1793]\end{array}\right.$} & $\begin{array}{l}\text { Obl, Obll, Tpal, Tpall, VI, VI(D), VII,VII(D), } \\
\text { bajo (Hay también un cuadernillo con } \\
\text { la tirana en partitura general y para } \\
\text { VI I, VII.) }\end{array}$ & $3 / 8$ & A dúo & $\begin{array}{l}8 \text { silabas } \\
\text { (TI-RI) }\end{array}$ & BHM, Mus 113-4 \\
\hline Poeta (EI)* & $\begin{array}{l}\text { LASERNA, } \\
\text { Blas de }\end{array}$ & 1791 & $\begin{array}{l}\text { Obl, Obll, Tpal, Tpall, VI, VI(D),VII,VII(D), } \\
\text { bajo }\end{array}$ & $6 / 8$ & A dúo & 8 sílabas & BHM, Mus 102-9 \\
\hline Porfiados (Los) & $\begin{array}{l}\text { LASERNA, } \\
\text { Blas de }\end{array}$ & {$[1780]$} & $\begin{array}{l}\text { Obl, Obll, Cl, Tpal, Tpall, VI, VI(D), VII, } \\
\text { VII(D), bajo, bajo(D) }\end{array}$ & $3 / 8$ & A dúo & 8 sílabas & BHM, Mus 158-9 \\
\hline $\begin{array}{l}\text { Provincias españolas } \\
\text { unidas por el placer (Las) }\end{array}$ & $\begin{array}{l}\text { LASERNA, } \\
\text { Blas de }\end{array}$ & 1789 & $\begin{array}{l}\text { Obl, Obll, Tpal-Tpal(D) (o clarín), Tpall- } \\
\text { Tpa II(D)(o clarín), VI, VI(2D),VII,VII(2D), } \\
\text { bajo, bajo(D) }\end{array}$ & $3 / 8$ & $\begin{array}{l}\text { A cuatro y } \\
\text { otros }\end{array}$ & $\begin{array}{l}8-6(12) \\
\text { sílabas }\end{array}$ & BHM, Mus 189-3 \\
\hline Razón y la moda (La) & $\begin{array}{l}\text { LASERNA, } \\
\text { Blas de }\end{array}$ & $\begin{array}{l}{[1783-} \\
1791]\end{array}$ & $\begin{array}{l}\text { Obl, Obll, Tpal, Tpall, VI, VI(D), VII,VII(D), } \\
\text { bajo }\end{array}$ & $3 / 8$ & $\begin{array}{l}\text { A dúo y } \\
\text { (trío). A } \\
\text { tres } \\
\end{array}$ & 8 sílabas & BHM, Mus 158-1 \\
\hline $\begin{array}{l}\text { Resurrección de la tirana } \\
(\mathrm{La})^{*}\end{array}$ & $\begin{array}{l}\text { LASERNA, } \\
\text { Blas de }\end{array}$ & 1781 & $\begin{array}{l}\text { Obl (sustituye Fl; está confundido } \\
\text { el compás, aunque la figuración } \\
\text { está correcta), Obll, Tpal, Tpall, VI, } \\
\text { VI(D),VII,VII(D),bajo }\end{array}$ & $3 / 8$ & A cuatro & $\begin{array}{l}8 \text { sílabas } \\
\text { (TI-RI) }\end{array}$ & BHM, Mus 151-3 \\
\hline Secreto de la Márquez (Los) & $\begin{array}{l}\text { LASERNA, } \\
\text { Blas de }\end{array}$ & 1795 & $\begin{array}{l}\text { Obl (sustituido por falutas), Obll } \\
\text { (sustituido por flautas), Tpal, Tpall, VI, } \\
\text { VI(D),VII,VII(D), contrabajo }\end{array}$ & $3 / 8$ & A solo & & $\begin{array}{l}\text { BHM, Mus [89- } \\
16] 80-16\end{array}$ \\
\hline Tercianista (EI) & $\begin{array}{l}\text { LASERNA, } \\
\text { Blas de }\end{array}$ & 30] & $\begin{array}{l}\text { Obl, Obll, Tpal, Tpall, VI, VI(D), VII,VII(D), } \\
\text { bajo }\end{array}$ & $3 / 8$ & A cuatro & 8 silc & is $148-5$ \\
\hline $\begin{array}{l}\text { Tornaboda de la gallega } \\
\text { (La) }\end{array}$ & $\begin{array}{l}\text { LASERNA, } \\
\text { Blas de }\end{array}$ & 1783 & Obl, Obll, VI, VI(D), VII, VII(D), bajo & $3 / 8$ & - & - & $\begin{array}{l}\text { BHM, Mus 162-5; } \\
\text { BNE, MC/3062/2 }\end{array}$ \\
\hline Trueque de los amantes (EI) & $\begin{array}{l}\text { LASERNA, } \\
\text { Blas de }\end{array}$ & \begin{tabular}{|l}
{$[1783-$} \\
$1791]$
\end{tabular} & Guitarras (2) & $3 / 8$ & A tres & 8 sílabas & BHM, Mus 147-8 \\
\hline
\end{tabular}


Revista de Humanidades, 41 (2020). p. 37-60. ISSN 1130-5029

\begin{tabular}{|c|c|c|c|c|c|c|c|}
\hline Trueque de los papeles (EI) & $\begin{array}{l}\text { LASERNA, } \\
\text { Blas de }\end{array}$ & 1785 & $\begin{array}{l}\text { Obl, Obll, Tpal, Tpall, VI, VI(D), VII,VII(D), } \\
\text { bajo }\end{array}$ & $3 / 8$ & A tres & 8-10 sílabas & BHM, Mus 130-4 \\
\hline Venida del soldado (La) & $\begin{array}{l}\text { LASERNA, } \\
\text { Blas de }\end{array}$ & \begin{tabular}{|l|}
{$[1787-$} \\
$1794 ?]$ \\
$(1805-$ \\
$1808)$ \\
\end{tabular} & $\begin{array}{l}\text { Obl, Obll, Tpal, Tpall, VI, VII, VII(D), bajo, } \\
\text { bajo(D) }\end{array}$ & $3 / 8$ & - & - & BHM, Mus 188-6 \\
\hline Buñolero (EI) & $\begin{array}{l}\text { MORAL, } \\
\text { Pablo del }\end{array}$ & 1799 & $\begin{array}{l}\text { Obl (sustituido por flautín), Obll } \\
\text { (sustituido por flautín), Tpal, Tpall, } \\
\text { VI, VI(D),VII,VII(D), contrabajo, } \\
\text { contrabajo(D) }\end{array}$ & $3 / 8$ & A cuatro & 8 sílabas & BHM, Mus 154-8 \\
\hline Burla del gallej[g]o (La) & \begin{tabular}{|l|} 
MORAL, \\
Pablo del
\end{tabular} & 1792 & $\begin{array}{l}\mathrm{VI}, \mathrm{VI}(\mathrm{D}), \mathrm{VII}, \mathrm{VII}(\mathrm{D}), \mathrm{Obl}, \mathrm{Obll}, \mathrm{Tpal}, \text { Tpall, } \\
\text { contrabajo }\end{array}$ & $3 / 8$ & A tres & $\begin{array}{l}\text { 8-10-12-13 } \\
\text { sílabas }\end{array}$ & BHM, Mus 171-3 \\
\hline $\begin{array}{l}\text { [Corte de mi vida, centro } \\
\text { de piedad] }\end{array}$ & $\begin{array}{l}\text { MORAL, } \\
\text { Pablo del }\end{array}$ & 1792 & Prueba. Moral & 0 & $\begin{array}{l}\text { Prueba. } \\
\text { Moral }\end{array}$ & - & BHM, Mus 89-11 \\
\hline De fuera vendrá & $\begin{array}{l}\text { MORAL, } \\
\text { Pablo del }\end{array}$ & 1797 & $\begin{array}{l}\mathrm{VI}, \mathrm{VI}(4 \mathrm{D}), \mathrm{VII}, \mathrm{VII}(4 \mathrm{D}) \text {, contrabajo, } \\
\text { contrabajo(D) }\end{array}$ & $3 / 8$ & A solo & 8-6-10 sílabas & BHM, Mus 137-6 \\
\hline Discordia (La) & \begin{tabular}{|l|} 
MORAL, \\
Pablo del \\
\end{tabular} & $\begin{array}{l}{[1790-} \\
1793] \\
\end{array}$ & $\begin{array}{l}\text { Obl, Obll, Tpal, Tpall, Vl, VI(D), VII, } \\
\text { contrabajo }\end{array}$ & $3 / 8$ & A dúo & 8 sílabas & BHM, Mus 108-8 \\
\hline $\begin{array}{l}\begin{array}{l}\text { Por qué de muchas cosas } \\
\text { (EI) }\end{array} \\
\end{array}$ & \begin{tabular}{|l|} 
MORAL, \\
Pablo del \\
\end{tabular} & 1788 & $\begin{array}{l}\text { Obl, Obll, Tpal, Tpall, VI, VI(D), VII, } \\
\text { contrabajo }\end{array}$ & $3 / 8$ & A tres & 8-5 silabas & BHM, Mus 184-3 \\
\hline Prueba (La) & $\begin{array}{l}\text { MORAL, } \\
\text { Pablo del }\end{array}$ & 1792 & $\begin{array}{l}\text { Obl (sustituido por flautín), Obll } \\
\text { (sustituido por flautín), Tpal, Tpall, VI, } \\
\text { VI(D), VII, VII (D), contrabajo } \\
\end{array}$ & $3 / 8$ & A solo & 8-12 sílabas & BHM, Mus 89-11 \\
\hline $\begin{array}{l}\text { Sillas del Prado y las } \\
\text { andaluzas (Las) }\end{array}$ & $\begin{array}{l}\text { MORAL, } \\
\text { Pablo del }\end{array}$ & 1791 & $\begin{array}{l}\text { Obl (sustituido por flauta), Obll } \\
\text { (sustituido por flauta), Tpal, Tpall, VI, } \\
\text { VI(D), VII, VII(D), contrabajo }\end{array}$ & $3 / 8$ & A cuatro & 8-4-5 sílabas & BHM, Mus 153-5 \\
\hline Tía burlada (La) & $\begin{array}{l}\text { MORAL, } \\
\text { Pablo del }\end{array}$ & 1791 & VI & $3 / 8$ & - & - & BHM, Mus $162-10$ \\
\hline $\begin{array}{l}\text { Gitana pobre y majo } \\
\text { enamorado }\end{array}$ & REMESSI & 1794 & \begin{tabular}{|l|} 
Obl (sustituido por otavines), Obll \\
(sustituidos por otavines), Tpal, Tpall, VI, \\
VI(D), VII, VII(D), contrabajo, contrabajo \\
(D)
\end{tabular} & $3 / 8$ & A dúo & 8-10 sílabas & BHM, Mus 115-3 \\
\hline Payo y la paya $(E I)^{*}$ & $\begin{array}{l}\text { ROSALES, } \\
\text { Antonio }\end{array}$ & $\begin{array}{l}{[1760-} \\
1789] \\
\end{array}$ & Obl, Obll, Tpal, Tpall, VI, VI(D), VII & $3 / 8$ & A dúo & 8 sílabas & BHM, Mus $116-10$ \\
\hline Chasco del abate (El) & $\begin{array}{l}\text { VALLEDOR, } \\
\text { Jacinto }\end{array}$ & 1785 & $\begin{array}{l}\text { Flauta I, Flauta II, Tpal, Tpall, VI, VI(D), VII, } \\
\text { VII(D), contrabajo }\end{array}$ & $3 / 8$ & A dúo & 8-10 silabas & BHM, Mus 141-6 \\
\hline Majos de rumbo (Los) & $\begin{array}{l}\text { VALLEDOR, } \\
\text { Jacinto }\end{array}$ & $\begin{array}{l}1802 \\
\text { Andioc, } \\
763 .\end{array}$ & $\begin{array}{l}\text { Ob I (sustituida por flauta), Ob II } \\
\text { (sustituida por FI), Cl, Tpal, Tpall, } \\
\text { VI, VI(D), VI (2a Tirana), VII, VII(D), } \\
\text { contrabajo, contrabajo }\end{array}$ & $3 / 8$ & A dúo & 8-10 sílabas & BHM, Mus 168-7 \\
\hline Compositor (El)* & Anónima & \begin{tabular}{|l|}
{$[1780-$} \\
$1794 ?]]$ \\
\end{tabular} & $\begin{array}{l}\text { Obl, Obll, Tpal, Tpall, VI, VI(D), VII, bajo } \\
(2 \mathrm{D})\end{array}$ & $3 / 8$ & A dúo & 8-12 sílabas & BHM, Mus 96-12 \\
\hline $\begin{array}{l}\text { Contrabandistas (Los) o } \\
\text { Cada uno con su suerte }\end{array}$ & $\begin{array}{l}\text { [Laserna] } \\
\text { Anónima }\end{array}$ & - & $\begin{array}{l}\text { Flautín I, Flautín II, CI I, Tpal, Tpall, VI, } \\
\text { VI(D), VII,VII(D),Vla, Fg, bajo, bajo(D), } \\
\text { tambor }\end{array}$ & $3 / 8$ & & - & BHM, Mus 158-7 \\
\hline (-1)Embusteros (Los) & Anónima & $\begin{array}{l}1783- \\
1791] \\
(1786) \\
\end{array}$ & $\begin{array}{l}\text { Obl, Obll, Tpal, Tpall, VI, VI(D), VII, } \\
\text { VII(D), bajo }\end{array}$ & $3 / 8$ & A tres & 8-10 sílabas & $\begin{array}{l}\text { BHM, Mus 147-5; } \\
\text { BNE, MC/3061/7 }\end{array}$ \\
\hline Erudita a la violeta (La) & Anónima & - & $\begin{array}{l}\text { Obl, Obll, Tpal, Tpall, VI, VI(D), VII, VII(D), } \\
\text { contrabajo y violón, contrabajo(D) }\end{array}$ & $3 / 8$ & - & - & BHM, Mus 178-6 \\
\hline Limera y el usía (La) & Anónima & $1765 ?$ & - & - & - & - & BHM, Mus 97-13 \\
\hline $\begin{array}{l}\text { Maestros de la Raboso } \\
\text { (Los), o El Trípili }\end{array}$ & Anónima & \begin{tabular}{|l|} 
Laserna \\
$1806)$
\end{tabular} & VI (Incompletas particellas) & $3 / 8$ & \begin{tabular}{|l|} 
A solo y \\
todos
\end{tabular} & 8 sílabas? & BHM, Mus 188-7 \\
\hline Miscelánea o Fin de fiesta & Anónima & $\begin{array}{l}1808 \\
\text { Carnaval }\end{array}$ & $\begin{array}{l}\text { Obl (sustituida por Flautín), Obll } \\
\text { (sustituida por Flautín), (Cl y Tpas?), VI, } \\
\text { VI(D), VII, VII(D), Vla, Fg, bajo, bajo(D) }\end{array}$ & $3 / 8$ & \begin{tabular}{|l|} 
A dúo \\
y todos \\
(hombres- \\
mujeres) \\
\end{tabular} & 8 sílabas & BHM, Mus 188-8 \\
\hline El tuno y calzetera & $\begin{array}{l}\text { LASERNA, } \\
\text { Blas de }\end{array}$ & 1782 & Ob I, Obll, Tpal, Tpall, VI, VII, bajo & $3 / 8$ & A dúo & 8 sílabas & BNE, MC/3056/6 \\
\hline
\end{tabular}


Tiranas y polacas en la tonadilla escénica... - Sara Navarro

\begin{tabular}{|c|c|c|c|c|c|c|c|}
\hline TÍTULO & AUTOR & AÑO & ORQUESTACIÓN POLACA & COMPÁS & PERSON. & $\begin{array}{l}\text { ESTRUCTURA } \\
\text { TEXTUAL }\end{array}$ & MSS \\
\hline $\begin{array}{l}\text { [Pobrecita, qué } \\
\text { congoja...! }]^{*}\end{array}$ & $\begin{array}{l}\text { LASERNA, } \\
\text { Blas de }\end{array}$ & 1790 & $\begin{array}{l}\text { Ob I, Obll, Tpal, Tpall, VI, VI(D), VII, } \\
\text { VII(D), bajo }\end{array}$ & $2 / 4$ & A solo & $\begin{array}{l}7,5,9,10 \\
\text { sílabas }\end{array}$ & \begin{tabular}{|l|} 
BHM, Mus 78-13 \\
{$[78-18]$}
\end{tabular} \\
\hline $\begin{array}{l}\text { Cada uno tiene su santo de } \\
\text { devoción }\end{array}$ & $\begin{array}{l}\text { MORAL, } \\
\text { Pablo del }\end{array}$ & 1800 & $\begin{array}{l}\text { Ob I, Obll, Tpal, Tpall, VI, VI(D), VII, VII(D), } \\
\text { contrabajo, contrabajo(D) }\end{array}$ & $3 / 4$ & A solo & 7 silabas & BHM, Mus 89-5 \\
\hline Casados por poderes (Los)* & $\begin{array}{l}\text { LASERNA, } \\
\text { Blas de }\end{array}$ & $\begin{array}{l}{[1784-} \\
1786 ?]\end{array}$ & \begin{tabular}{|l|} 
Ob I(sustituido por flautin), Obll \\
(sustituido por flautín), Tpal, Tpall,Vl, \\
VI(D), VII, VII(D), Fgl, Fgll, bajo, bajo(D) \\
\end{tabular} & $3 / 8$ & A solo & 7 silabas & BHM, Mus 168-9 \\
\hline Crítica de la petimetra $(\mathrm{La})^{*}$ & $\begin{array}{l}\text { LASERNA, } \\
\text { Blas de }\end{array}$ & 1788 & $\begin{array}{l}\text { Ob I, Obll, Tpal, Tpall, VI, VI(D), VII, } \\
\text { VII(D), bajo }\end{array}$ & $2 / 4$ & A solo & $\begin{array}{l}6-7-8-10 \\
\text { silabas }\end{array}$ & BHM, Mus 80-1 \\
\hline Enredo de Tadeo (El)* & $\begin{array}{l}\text { ESTEVE, } \\
\text { Pablo }\end{array}$ & \begin{tabular}{|l|}
$1780]$ \\
patitura \\
general \\
\end{tabular} & $\begin{array}{l}\text { Ob I, Obll, Tpal, Tpall, Vl, VI(D), VII, } \\
\text { VII(D), bajo }\end{array}$ & $3 / 8$ & A solo & 6 (7) sílabas & $\begin{array}{l}\text { BHM, Mus } \\
150-15\end{array}$ \\
\hline Feria y jota $(\mathrm{La})^{*}$ & Anónima & & $\begin{array}{l}\text { Tpal, Tpall, VI, VI (D), VII, VII(D), } \\
\text { contrabajo }\end{array}$ & $6 / 8$ & A solo & $5-6-7-8$ sílabas & BHM, Mus 75-21 \\
\hline $\begin{array}{l}\text { Fin de fiesta de las } \\
\text { funciones Reales }\end{array}$ & $\begin{array}{l}\text { LASERNA, } \\
\text { Blas de }\end{array}$ & 1803 & En tiranas & $2 / 4$ & A solo & 7 silabas & \begin{tabular}{|l|} 
BHM, Mus \\
$642-10$ \\
\end{tabular} \\
\hline Intríngulis (EI) & $\begin{array}{l}\text { MORAL, } \\
\text { Pablo del }\end{array}$ & 1800 & $\begin{array}{l}\text { Obl, Obll, Clarinete, Tpal, Tpall, Vl, } \\
\text { VI(D), VII, VII(D), Vla, Fg, contrabajo, } \\
\text { contrabajo y violón(D) }\end{array}$ & $3 / 4$ & A solo & 8 silabas & BHM, Mus 87-7 \\
\hline Ocasión hace el ladrón (La) & $\begin{array}{l}\text { LASERNA, } \\
\text { Blas de }\end{array}$ & (1766) & $\begin{array}{l}\text { Ob I, Obll, Tpal, Tpall, Vl, VI(D), VII, } \\
\text { VII(D), bajo }\end{array}$ & $3 / 4$ & A solo & 7 silabas & BHM, Mus 81-7 \\
\hline Petardista (El) & $\begin{array}{l}\text { MORAL, } \\
\text { Pablo del }\end{array}$ & (1804) & \begin{tabular}{|l|} 
Obl, Obll, Clarinete, Tpal, Tpall, VI, VI(D), \\
VII, VII(D), Vla, Fg, bajo, contrabajo(D)
\end{tabular} & $3 / 4$ & A tres & 7 silabas & BHM, Mus 130-3 \\
\hline Retratos y los billetes (Los) & $\begin{array}{l}\text { MORAL, } \\
\text { Pablo del }\end{array}$ & 1799 & - & $3 / 4$ & A tres & 7 silabas & \begin{tabular}{|l|} 
BHM, Mus \\
$145-17$
\end{tabular} \\
\hline Secretos (Los) & $\begin{array}{l}\text { MORAL, } \\
\text { Pablo del }\end{array}$ & 1800 & $\begin{array}{l}\text { Ob I, Obll, Tpal, Tpall, VI, VI(D), VII, VII(D), } \\
\text { contrabajo, contrabajo(D) }\end{array}$ & $3 / 8$ & A solo & 6 silabas & BHM, Mus 88-2 \\
\hline Sin título (La) & $\begin{array}{l}\text { MORAL, } \\
\text { Pablo del }\end{array}$ & 1797 & $\begin{array}{l}\text { Ob I, Obll, Tpal, Tpall, VI, VI(D), VII, VII(D), } \\
\text { contrabajo y violón }\end{array}$ & $3 / 4$ & A solo & 8 silabas & BHM, Mus 89-2 \\
\hline Visita del Nuevo (La) & $\begin{array}{l}\text { LASERNA, } \\
\text { Blas de }\end{array}$ & \begin{tabular}{|l|}
$1794)$ \\
partitura \\
general
\end{tabular} & $\begin{array}{l}\text { Ob I, Obll, Tpal, Tpall, VI, VI(D), VII, VII(D), } \\
\text { contrabajo }\end{array}$ & $3 / 4$ & A solo & 7 silabas & BHM, Mus 134-7 \\
\hline La Paxarera y cazadores* & $\begin{array}{l}\text { LASERNA, } \\
\text { Blas de }\end{array}$ & 1779 & $\begin{array}{l}\text { Ob I, Obll, Tpal, Tpall, VI, VI(D), VII, VII(D), } \\
\text { contrabajo }\end{array}$ & $3 / 4$ & A tres & $7-5$ silabas & BNE, MC/3058/4 \\
\hline \begin{tabular}{|l|} 
Las tonadillas \\
interrumpidas*
\end{tabular} & $\begin{array}{l}\text { LASERNA, } \\
\text { Blas de }\end{array}$ & 1785 & $\begin{array}{l}\text { Tpal, Tpall, VI, VI(D), VII, VII(D), } \\
\text { contrabajo }\end{array}$ & $2 / 4$ & A dúo & 6 silabas & BNE, MC/3057/6 \\
\hline La temerosa* & $\begin{array}{l}\text { LASERNA, } \\
\text { Blas de }\end{array}$ & 1787 & $\begin{array}{l}\text { Tpal, Tpall, VI, VI(D), VII, VII(D), } \\
\text { contrabajo }\end{array}$ & $2 / 4$ & A dúo & $8-6$ silabas & \begin{tabular}{|l|}
$\mathrm{BNE}$, \\
$\mathrm{MC} / 3057 / 12$
\end{tabular} \\
\hline La tímida* & $\begin{array}{l}\text { LASERNA, } \\
\text { Blas de }\end{array}$ & 1799 & $\begin{array}{l}\text { Ob I, Obll, Tpal, Tpall, VI, VI(D), VII, VII(D), } \\
\text { contrabajo }\end{array}$ & $3 / 4$ & A solo & 5 silabas & BNE, M/3583 \\
\hline La Yrónica* & $\begin{array}{l}\text { LASERNA, } \\
\text { Blas de }\end{array}$ & 1786 & $\begin{array}{l}\text { Ob I, Obll, Tpal, Tpall, VI, VI(D), VII, VII(D), } \\
\text { contrabajo }\end{array}$ & $2 / 4$ & A solo & $7-5$ silabas & BNE, MC/3052/1 \\
\hline Sin embargo caballeros* & $\begin{array}{l}\text { LASERNA, } \\
\text { Blas de }\end{array}$ & 1779 & $\begin{array}{l}\text { Ob I, Obll, Tpal, Tpall, VI, VI(D), VII, VII(D), } \\
\text { contrabajo }\end{array}$ & $3 / 4$ & A solo & 6 silabas & BNE, MC/3053/3 \\
\hline $\begin{array}{l}\text { Tonadilla á solo con } \\
\text { violines* }\end{array}$ & $\begin{array}{l}\text { LASERNA, } \\
\text { Blas de }\end{array}$ & - & $\mathrm{VI}, \mathrm{VI}(\mathrm{D}), \mathrm{VII}, \mathrm{VII}(\mathrm{D})$, contrabajo & $6 / 8$ & A solo & 10 silabas & BNE, MC/3052/9 \\
\hline
\end{tabular}

*Estas tonadillas escénicas contienen los términos tirana y polaca respectivamente, aunque el autor no especifica que se trate de dicho número. 